hep-ph/0107309

ANL-HEP-01-057

\title{
QCD Factorized Drell-Yan Cross Section at Large Transverse Momentum
}

\author{
Edmond L. Berger ${ }^{a}$, Jianwei Qiu ${ }^{b}$ and Xiaofei Zhang ${ }^{b}$ \\ ${ }^{a}$ Division of High Energy Physics, Argonne National Laboratory \\ Argonne, Illinois 60439, USA \\ ${ }^{b}$ Department of Physics and Astronomy, Iowa State University \\ Ames, Iowa 50011, USA
}

(July 30, 2001)

\begin{abstract}
We derive a new factorization formula in perturbative quantum chromodynamics for the Drell-Yan massive lepton-pair cross section as a function of the transverse momentum $Q_{T}$ of the pair. When $Q_{T}$ is much larger than the pair's invariant mass $Q$, this factorization formula systematically resums the logarithmic contributions of the type $\alpha_{s}^{m} \ln ^{m}\left(Q_{T}^{2} / Q^{2}\right)$ to all orders in the strong coupling $\alpha_{s}$. When $Q_{T} \sim Q$, our formula yields the same Drell-Yan cross section as conventional fixed order QCD perturbation theory. We show that resummation is important when the collision energy $\sqrt{S}$ is large enough and $Q_{T} \gg Q$, and we argue that perturbative expansions are more stable and reliable in terms of the modified factorization formula.
\end{abstract}

PACS Numbers: 12.38.Bx, 12.38.cy, 13.85.Qk, 14.70.Bh

Typeset using REVTEX 


\section{INTRODUCTION}

The study of massive lepton-pair production in hadronic collisions (the Drell Yan process) has been a valuable pursuit for many years [1]. The process is an excellent laboratory for theoretical and experimental investigations of strong interaction dynamics, and it is a channel for discovery of quarkonium states and intermediate vector bosons. In the Drell Yan process, the massive lepton-pair is produced via the decay of an intermediate virtual photon, $\gamma^{*}$. Within the context of perturbative quantum chromodynamics (QCD), the Drell-Yan cross section in a collision between hadrons $A$ and $B, A\left(P_{A}\right)+B\left(P_{B}\right) \rightarrow \gamma^{*}(\rightarrow l \bar{l}(Q))+X$, can be expressed in terms of the cross section for production of an unpolarized virtual photon of the same invariant mass [2],

$$
\frac{d \sigma_{A B \rightarrow \ell^{+} \ell^{-}(Q) X}}{d Q^{2} d Q_{T}^{2} d y}=\left(\frac{\alpha_{e m}}{3 \pi Q^{2}}\right) \frac{d \sigma_{A B \rightarrow \gamma^{*}(Q) X}}{d Q_{T}^{2} d y} .
$$

The variables $Q, Q_{T}$, and $y$ are the invariant mass, transverse momentum, and rapidity of the pair. Symbol $X$ stands for an inclusive sum over final states that recoil against the virtual photon. An integration has been performed over the angular distribution in the lepton-pair rest frame. Because the leptons can be detected and measured without restrictions, massive lepton-pair production as well as inclusive virtual photon production defined in Eq. (1) are entirely inclusive.

Precise knowledge of the gluon parton distribution in nucleons is critical for reliable predictions of the signals and backgrounds for many important reactions studied at the Fermilab Tevatron and CERN Large Hadron Collider (LHC). It was pointed out recently that the transverse momentum distribution of massive lepton-pairs produced in hadronic collisions is an advantageous source of constraints on the gluon distribution [2], free from the experimental and theoretical complications of photon isolation that beset studies of prompt photon production [3, 田. Other than the difference between a virtual and a real photon, the Drell-Yan process and prompt photon production share the same partonic subprocesses. Similar to prompt photon production, the lowest-order virtual photon "Compton" subprocess: $g+q \rightarrow \gamma^{*}+q$ dominates the $Q_{T}$ distribution when $Q_{T}>Q / 2$, and the next-to-leading order contributions preserve the fact that the $Q_{T}$ distributions are dominated by gluon initiated partonic subprocesses [2].

If both physically measured quantities $Q$ and $Q_{T}$ are large, the cross section for lepton pairs of invariant mass $Q$ and transverse momentum $Q_{T}$ can be factored systematically in QCD perturbation theory and expressed as [5,6]

$$
\frac{d \sigma_{A B \rightarrow \gamma^{*}(Q) X}}{d Q_{T}^{2} d y}=\sum_{a, b} \int d x_{1} \phi_{a / A}\left(x_{1}, \mu\right) \int d x_{2} \phi_{b / B}\left(x_{2}, \mu\right) \frac{d \hat{\sigma}_{a b \rightarrow \gamma^{*}(Q) X}}{d Q_{T}^{2} d y}\left(x_{1}, x_{2}, Q, Q_{T}, y ; \mu\right) .
$$

The sum $\sum_{a, b}$ runs over all parton flavors; $\phi_{a / A}$ and $\phi_{b / B}$ are normal parton distributions; and $\mu$ is the renormalization and the factorization scale. The function $d \hat{\sigma}_{a b \rightarrow \gamma^{*}(Q) X} / d Q_{T}^{2} d y$ in Eq. (2) represents the short-distance physics of the collision and is calculable perturbatively in terms of a power series in $\alpha_{s}(\mu)$. The leading order and next-to-leading order contributions are available [2].7]. The scale $\mu$ is of the order of the energy exchange in the reaction, $\mu \sim \sqrt{Q^{2}+Q_{T}^{2}}$ 
There is a phase space penalty associated with the finite mass of the virtual photon, and the Drell-Yan factor $\alpha_{e m} /\left(3 \pi Q^{2}\right)<10^{-3} / Q^{2}$ renders the production rates for massive lepton pairs small at large values of $Q$ and $Q_{T}$. In order to enhance the Drell-Yan cross section while keeping the dominance of the gluon initiated subprocesses, it is useful to study lepton pairs with low invariant mass and relatively large transverse momenta [2]. With the large transverse momentum $Q_{T}$ setting the hard scale of the collision, the invariant mass of the virtual photon $Q$ can be small, as long as the process can be identified experimentally, and the numerical value $Q \gg \Lambda_{\mathrm{QCD}}$. For example, the cross section for Drell-Yan production was measured by the CERN UA1 Collaboration [8] for virtual photon mass $Q \in\left[2 m_{\mu}, 2.5\right] \mathrm{GeV}$.

When $Q_{T}$ is very different from $Q$ while both are much larger than $\Lambda_{\mathrm{QCD}}$, the calculation of massive lepton-pair production becomes a two-scale problem in QCD perturbation theory. The corresponding short-distance partonic parts, calculated in conventional fixed-order QCD perturbation theory, include potentially large terms proportional to the logarithm of the ratio of these two physical scales. As a result, the higher-order corrections in powers of $\alpha_{s}$ are not necessarily small. The ratio $\sigma^{N L O} / \sigma^{L O}\left[\propto \alpha_{s} \times\right.$ (large logarithms)] can be of order 1 , and the convergence of the conventional perturbative expansion in powers of $\alpha_{s}$ is possibly impaired.

When $Q_{T}^{2} \ll Q^{2}$, the Drell-Yan (or $W^{ \pm}$and $Z$ ) transverse momentum distributions calculated in fixed-order QCD perturbation theory are known not to be reliable [9]. After allorders resummation of the large $\ln \left(Q^{2} / Q_{T}^{2}\right)$ terms is performed, predictions for the transverse momentum distributions become consistent with data for $Q_{T}^{2} \ll Q^{2}$ [10,11]. Similarly, when $Q_{T}^{2} \gg Q^{2}$, the region of phase space of interest in this paper, the perturbatively calculated short-distance partonic parts, $d \hat{\sigma}_{a b \rightarrow \gamma^{*}(Q) X} / d Q_{T}^{2} d y$ in Eq. (2), receive one power of the logarithm $\ln \left(Q_{T}^{2} / Q^{2}\right)$ at every order of $\alpha_{s}$ beyond the leading order. At sufficiently large $Q_{T}$, the coefficients of the perturbative expansion in $\alpha_{s}$ will have large logarithmic terms, and these high order corrections may not be small. In order to derive reliable QCD predictions, resummation of the logarithmic terms $\ln ^{m}\left(Q_{T}^{2} / Q^{2}\right)$ must be considered.

The purpose of this paper is to modify the factorization formula in Eq. (2) so that resummation of the logarithmic contributions can be included naturally when $Q_{T}^{2} \gg Q^{2}$. At the same time, the modified factorization formula should remain effectively the same as the conventional factorization formula in Eq. (21) when $Q_{T}^{2} \sim Q^{2}$.

In the next section, we review the general structure of the Drell-Yan cross section, and we identify where the large logarithms arise when $Q_{T}^{2} \gg Q^{2} \gg \Lambda_{\mathrm{QCD}}^{2}$. We show that when $Q^{2}$ is fixed and $Q^{2} / Q_{T}^{2} \rightarrow 0$, the Drell-Yan cross section behaves similarly to the cross section for prompt real photon production [2]. The large logarithmic contributions to the Drell-Yan cross section at high $Q_{T}$ come from partonic processes that fall into the two-stage generic pattern of fragmentation contributions: (1) short-distance production of a parton of momentum $p_{c}$, and (2) fragmentation of this parton into the observed virtual photon.

In Sec. [II], we show that the $\ln ^{m}\left(Q_{T}^{2} / Q^{2}\right)$ logarithmic contributions to the Drell-Yan cross section can be resummed systematically to all orders in $\alpha_{s}$. We demonstrate that these resummed logarithmic contributions have the same factored form as those for single hadron production at large transverse momentum, with the parton-to-hadron fragmentation functions replaced by the fragmentation functions for a parton to a virtual photon of invariant mass $Q$. As for single hadron production, the short-distance production of the parton is evaluated at a single hard scale $\left(\sim Q_{T}\right)$, and it is calculable perturbatively in a power series 
of $\alpha_{s}$. However, unlike the parton-to-hadron fragmentation functions, the parton-to-virtualphoton fragmentation functions are calculable perturbatively if $Q^{2} \gg \Lambda_{\mathrm{QCD}}^{2}$ [12].

In addition to the logarithmic contributions, the Drell-Yan cross section includes large non-logarithmic contributions, in particular, the leading order contributions, referred to often as direct contributions. In Sec. IV, we derive our modified factorization formula for massive lepton-pair production, Eq. (35), in which both logarithmic and non-logarithmic contributions are fully included. This modified factorization formula agrees with the conventional fixed-order QCD expression when $Q_{T}^{2} \sim Q^{2}$ (or when the logarithmic contributions are less important). We show that at the next-to-leading order, the difference between the modified factorization formula and the conventional factorization formula is completely determined by QCD evolution of the virtual photon fragmentation functions. Our modified factorization formalism reorganizes the single perturbative expansion of conventional QCD factorization into two perturbative expansions plus the perturbatively calculated parton-tovirtual photon fragmentation functions. The main advantage of this reorganization is that the new perturbative expansions are evaluated at a single hard scale and are free of large logarithmic terms for $Q_{T} \geq Q$.

In Sec. Q , we present our predictions for the cross sections for massive lepton-pair production at energies of interest for experiments at the Fermilab Tevatron, Brookhaven's RHIC, and the CERN Large Hadron Collider. We include both leading order and next-to-leading order direct short-distance contributions and the resummed logarithmic contributions. The resummed large logarithmic contributions change the shape of the predicted $Q_{T}$-spectrum of the Drell-Yan cross section but, at the order in perturbation theory at which we work, they have only a modest effect on the normalization. We confirm that after the large logarithmic terms are resummed to all orders in $\alpha_{s}$ the Drell-Yan cross section at large $Q_{T}$ remains an excellent source of contraints on the gluon parton density. Our conclusions are summarized in Sec. V1.

\section{MASSIVE LEPTON PAIR PRODUCTION AT FIXED ORDER}

In hadronic collisions, massive lepton pair production proceeds through partonic hardscattering processes involving initial-state quarks and gluons. If the lepton pair's invariant mass $Q$ and its transverse momentum $Q_{T}$ are both much larger than $\Lambda_{\mathrm{QCD}}$, the partonic hardscattering at a distance scale between $O(1 / Q)$ and $O\left(1 / Q_{T}\right)$ can be systematically factored from the physics at the scale of hadron wave functions, $O\left(1 / \Lambda_{\mathrm{QCD}}\right)$. In this situation, the cross section can be expressed in the factored form of Eq. (2). Corrections to the expression in Eq. (2) are suppressed by powers of $\Lambda_{\mathrm{QCD}}^{2} / Q^{2}$ or $\Lambda_{\mathrm{QCD}}^{2} / Q_{T}^{2}$. The predictive power of Eq. (22) relies on the universality of the parton distributions and the reliability of the partonic cross sections.

The short-distance partonic cross sections, $d \hat{\sigma}_{a b \rightarrow \gamma^{*}(Q) X} / d Q_{T}^{2} d y$ in Eq. (国), can be calculated in principle order by order in QCD perturbation theory in a power series of the strong coupling $\alpha_{s}$,

$$
\frac{d \hat{\sigma}_{a b \rightarrow \gamma^{*}(Q) X}}{d Q_{T}^{2} d y}=\sum_{n=0} H_{a b \rightarrow \gamma^{*} X}^{(n)}\left(x_{1}, x_{2}, Q, Q_{T}, y ; \mu\right)\left(\frac{\alpha_{s}(\mu)}{2 \pi}\right)^{n} .
$$


The reliability of QCD perturbative calculations depends on the behavior of the coefficient functions $H_{a b \rightarrow \gamma^{*} X}^{(n)}$ in Eq. (3).

At lowest-order, $O\left(\alpha_{s}^{0}\right)$, the only partonic subprocess for virtual photon production is $q+\bar{q} \rightarrow \gamma^{*}$. The incoming partons are assumed to be collinear to their respective incoming hadrons if power suppressed corrections are neglected. Therefore, the lowest order coefficient function, $H_{q \bar{q} \rightarrow \gamma^{*} X}^{(0)} \propto \delta\left(Q_{T}\right)$, vanishes if $Q_{T} \neq 0$.

At order $O\left(\alpha_{s}\right)$, both quark-antiquark annihilation, $q+\bar{q} \rightarrow \gamma^{*}+g$, and "Compton", $g+q \rightarrow \gamma^{*}+q$, subprocesses contribute to the Drell-Yan cross section, with the recoil of the final-state parton balancing the transverse momentum of the lepton pair. These partonic subprocesses, known as the $2 \rightarrow 2$ subprocesses, are shown in Figs. 1(a) and (b). They are often referred as the leading order (LO) contributions to the Drell-Yan cross section with finite transverse momentum. The corresponding leading order coefficient functions are

$$
H_{a b \rightarrow \gamma^{*} X}^{(1)}=e_{q}^{2} \frac{\pi}{2 x_{1} x_{2} S}\left|\frac{1}{g_{s}} \bar{M}_{a b \rightarrow \gamma^{*} X}\right|^{2}\left(8 \pi^{2}\right) \delta\left(\left(x_{1} P_{A}+x_{2} P_{B}-Q\right)^{2}\right)\left(\frac{\alpha_{e m}}{2 \pi}\right) .
$$

The incoming parton flavors " $a b$ " can be either $q \bar{q}$ for the quark-antiquark annihilation or $g q$ for the Compton subprocess, and $e_{q}$ is quark's fractional charge. In Eq. (44), $g_{s}$ and $\alpha_{e m}$ are the strong coupling constant and the fine structure constant of QED, respectively; $P_{A}$ and $P_{B}$ are the momenta of the colliding hadrons, and $S=\left(P_{A}+P_{B}\right)^{2}$ is the square of the total collision energy. The expressions $\left|\bar{M}_{a b \rightarrow \gamma^{*} X}\right|^{2}$ in Eq. (四) are the squares of the matrix elements of the partonic subprocesses shown in Fig. 1, summed (averaged) over the colors and spins of the final-state (initial-state) partons. They are available in Ref. [13]. As long as $Q_{T}$ is large, the LO coefficient functions in Eq. (四) are well-behaved, even when $Q^{2} \rightarrow 0$.

The calculation of the perturbative coefficient functions at order $O\left(\alpha_{s}^{2}\right)$, known as the next-to-leading order (NLO) contribution, involve all $2 \rightarrow 3$ partonic subprocesses with the virtual photon in the final-state as well as the $2 \rightarrow 2$ diagrams in Fig. 1 1 with one-loop corrections. After renormalization, the loop momentum integrations for the $2 \rightarrow 2$ diagrams at order $O\left(\alpha_{s}^{2}\right)$ yield renormalization scale $(\mu)$ dependence and logarithmic terms in the coefficient functions $H_{a b \rightarrow \gamma^{*} X}^{(2)}$. Integration over the phase space of the extra parton in the final-state of the $2 \rightarrow 3$ subprocesses leads to a collinear divergence when this parton is collinear to either incoming parton. QCD factorization and subtraction of the collinear divergence results in factorization scale $\left(\mu_{f}\right)$ dependence and logarithmic terms in the coefficient functions at this order. Consequently, the coefficient functions $H_{a b \rightarrow \gamma^{*} X}^{(2)}$ display logarithmic dependence on the ratios of the following momentum scales: $\mu, \mu_{f}, Q_{T}$, and $Q$ [2.,7].

Since we are interested in identifying the logarithms of the ratio $Q_{T}^{2} / Q^{2}$, with $Q_{T}^{2} \gg Q^{2}$, we concentrate on the part of the Drell-Yan cross section that diverges when $\ln \left(Q_{T}^{2} / Q^{2}\right) \rightarrow$ $\infty$ with $Q^{2} \gg \Lambda_{\mathrm{QCD}}^{2}$. According to the QCD factorization theorem, the perturbatively calculated partonic cross sections $d \hat{\sigma}_{a b \rightarrow \gamma^{*}(Q) X} / d Q_{T}^{2} d y$ in Eq. (2) should be analytic functions of $Q_{T}^{2}$ and $Q^{2}$. Therefore, we expect the logarithmic behavior of the Drell-Yan cross section as $\ln \left(Q_{T}^{2} / Q^{2}\right) \rightarrow \infty$ with $Q^{2} \gg \Lambda_{\mathrm{QCD}}^{2}$ fixed to be connected closely to logarithmic divergences associated with the massless photon $\left(Q^{2}=0\right)$ in the case of prompt real photon production.

Other than the non-vanishing invariant mass, production of a virtual photon and a real photon share the same parton-level Feynman diagrams. However, the QCD factorization formula for production of a real photon $\left(Q^{2}=0\right)$ is different from that in Eq. (2), 


$$
\frac{d \sigma_{A B \rightarrow \gamma X}}{d Q_{T}^{2} d y}=\sum_{a, b} \int d x_{1} \phi_{a / A}\left(x_{1}, \mu\right) \int d x_{2} \phi_{b / B}\left(x_{2}, \mu\right)\left[\frac{d \hat{\sigma}_{a b \rightarrow \gamma X}^{(D i r)}}{d Q_{T}^{2} d y}+\frac{d \hat{\sigma}_{a b \rightarrow \gamma X}^{(F)}}{d Q_{T}^{2} d y}\right],
$$

where $d \hat{\sigma}_{a b \rightarrow \gamma X}^{(D i r)} / d Q_{T}^{2} d y$ represents the direct production of the real photon at a short-distance scale of $O\left(1 / Q_{T}\right)$; and

$$
\frac{d \hat{\sigma}_{a b \rightarrow \gamma X}^{(F)}}{d Q_{T}^{2} d y}=\sum_{c} \int \frac{d z}{z^{2}} \frac{d \hat{\sigma}_{a b \rightarrow c X}}{d p_{c_{T}}^{2} d y}\left(p_{c}=\frac{Q}{z}, \mu_{F}^{2}\right) D_{c \rightarrow \gamma X}\left(z, \mu_{F}^{2}\right)
$$

is the fragmentation contribution to the prompt photon cross section. The function $d \hat{\sigma}_{a b \rightarrow c X} / d p_{c_{T}}^{2} d y$ in Eq. (6) represents short-distance production of a parton of flavor $c$, and $D_{c \rightarrow \gamma X}\left(z, \mu_{F}^{2}\right)$ is a fragmentation function for parton $c$ to fragment into a real photon with photon momentum $Q=z p_{c} ; \mu_{F}$ is the fragmentation scale.

The fragmentation contribution arises because there are collinear singularities associated with the region of phase space in which the real photon is parallel to one or more of the final-state partons [13,14. Because of the real photon is massless, the parent parton, which fragments into the real photon and other collinear partons, can propagate for a long time. Consequently, quantum interference between the production of the parent parton and the physics associated with the fragmentation (or decay) of the parton is suppressed. Therefore, the fragmentation contribution to prompt photon production can be further factored as in Eq. (6). Because the transverse momentum $Q_{T}$ is large, all logarithmic collinear divergences associated with the massless photon arise from final-state partons that are parallel to the observed real photon. Such logarithmic divergences are all absorbed into the fragmentation functions. These functions are nonperturbative in nature.

Unlike prompt photon production, it is not necessary to introduce fragmentation functions to absorb final-state collinear singularities for the Drell-Yan cross section. Because the photon is off-shell, its large invariant mass $Q$ regulates the singularity. This finite mass regularization leads to a logarithmic dependence of the Drell-Yan cross section on the invariant mass of the virtual photon $Q$. If $Q$ is large enough and $Q_{T}$, the only other physically observed momentum scale, is not too large, the logarithmic terms $\ln ^{m}\left(Q_{T}^{2} / Q^{2}\right)$ are small, and no resummation of the logarithms is necessary for a reliable prediction of the cross section.

When $Q^{2}$ is chosen to be small, so as to enhance the Drell-Yan cross section, and when the collision energy $\sqrt{S}$ and $Q_{T}$ become large, it is necessary to examine the size of the final-state logarithmic contributions and ascertain whether resummation of these logarithmic terms is warranted.

The explicit form of NLO contributions to the Drell-Yan cross section from a $2 \rightarrow 3$ partonic subprocess $q+\bar{q} \rightarrow \gamma^{*}(Q)+q^{\prime}+\bar{q}^{\prime}$ [2] provides an example of the logarithmic terms under discussion. The relevant Feynman diagrams are shown in Fig. 2(a) and 2(b) for initial- and final-state photon radiation. Since we are interested mainly in the structure of the final state in the region where a photon becomes collinear to a quark, this subprocess is typical of the generic $2 \rightarrow 3$ subprocess in Fig. 3, and conclusions drawn from it can applied to other subprocesses such as $q g$ and $q q^{\prime}$ scattering. For the phase space integrals of the diagrams in Fig. 2, we use the exact forms in Ref. [15]. The logarithmic contributions have the form

$$
\ln \left[\frac{s+Q^{2}-s_{2}+\lambda}{s+Q^{2}-s_{2}-\lambda}\right] g\left(s, t, u, Q^{2}\right) .
$$


The function $g\left(s, t, u, Q^{2}\right)$ is given in Ref. [2]. It is well-behaved as $Q^{2} \rightarrow 0$. The parton-level Mandelstam variables $s, t, u$ are defined as $s=\left(p_{1}+p_{2}\right)^{2}, t=\left(p_{1}-Q\right)^{2}$, and $u=\left(p_{2}-Q\right)^{2}$. The function $\lambda=\sqrt{(t+u)^{2}-4 Q^{2} s_{2}}$, with $s_{2} \equiv\left(p_{1}+p_{2}-Q\right)^{2}=s+t+u-Q^{2} ; s_{2}$ is the square of the invariant mass of the two final-state partons that recoil against $Q$.

In the limit $Q^{2} \ll|t+u|$, the generic logarithm from the splitting of a quark into a photon can be approximated as

$$
\begin{aligned}
\ln \left[\frac{s+Q^{2}-s_{2}+\lambda}{s+Q^{2}-s_{2}-\lambda}\right] & \rightarrow \ln \left[\frac{2 Q^{2}\left(s-Q^{2}\right) /(-(t+u))}{2(-(t+u))}\right] \\
& \rightarrow-\ln \left[\frac{-(t+u)}{Q^{2}}\right]
\end{aligned}
$$

To derive the second line we use $Q^{2} \ll|t+u|$ and $s \sim|t+u|$. Since $|t+u| \sim O\left(Q_{T}^{2}+Q^{2}\right)$, the logarithm takes the form $\ln \left[\left(Q_{T}^{2}+Q^{2}\right) / Q^{2}\right] \sim \ln \left[Q_{T}^{2} / Q^{2}\right]$, and the limit of $Q^{2} \ll|t+u|$ is effectively the same as $Q^{2} \ll Q_{T}^{2}$. The logarithmic contributions to the Drell-Yan cross section become more important when $Q^{2}$ is chosen to be small.

In principle, we can resum these large but finite logarithms into parton-to-virtual-photon fragmentation functions, just as the logarithmic divergences in real photon production are resummed into real-photon fragmentation functions. Because the logarithms in the Drell-Yan case are finite, and $Q^{2}$ is much larger than $\Lambda_{\mathrm{QCD}}^{2}$, the parton-to-virtual-photon fragmentation functions should be calculable perturbatively [12].

\section{FRAGMENTATION CONTRIBUTIONS TO THE DRELL-YAN CROSS SECTION}

In this section, we derive the resummed logarithmic contributions to the Drell-Yan cross section when $Q_{T}^{2} \gg Q^{2}$. Since we are interested mainly in the cross section at low $Q^{2}$, we ignore contributions from the intermediate vector boson $Z$.

\section{A. Logarithmic Contributions to the Drell-Yan Cross Section at Large $Q_{T}$}

As demonstrated in the last section, the Drell-Yan cross section at large transverse momentum $Q_{T}$ receives potentially large logarithmic terms $\ln ^{m}\left(Q_{T}^{2} / Q^{2}\right)$ from the part of phase space in which the virtual photon is almost collinear to one or more final-state partons. Since at least one final-state parton is needed to balance the virtual photon's transverse momentum, the logarithmic contributions can arise only at NLO and beyond.

Because of the logarithms, the coefficient functions in Eq. (3) might be large, and resummation of the logarithmic contributions might be needed. As long as $Q^{2}$ is much larger than $\Lambda_{\mathrm{QCD}}^{2}$, all coefficient functions in Eq. (3) are calculable in principle order by order in QCD

perturbation theory. Therefore, resummation of the logarithms $\ln ^{m}\left(Q_{T}^{2} / Q^{2}\right)$ is actually a reorganization of the perturbative expansion in Eq. (3), such that all coefficient functions in the reorganized perturbative expansions are evaluated at a single hard scale and free of any large logarithms. 
The energy exchange in the hard collision is of the order of $\sqrt{Q_{T}^{2}+Q^{2}} \approx Q_{T}+O\left(Q^{2} / Q_{T}^{2}\right)$. When $Q_{T}^{2} \gg Q^{2}$, the partonic hard collision should not be sensitive to the scale $Q^{2}$ at which the virtual photon is produced. If we neglect power corrections of the order $Q^{2} / Q_{T}^{2}$, the partonic hard collisions are effectively independent of $Q^{2}$, except for the logarithmic dependence $\ln ^{m}\left(Q_{T}^{2} / Q^{2}\right)$ from the final-state bremsstrahlung production of the virtual photon. Therefore, other than the appearance of the virtual photon's mass $Q$ to regulate the finalstate collinear divergences, the potentially large logarithmic contributions at large $Q_{T}$ have the same structure as the fragmentation contributions to prompt real photon production. They can be separated into two stages: (1) production of a parton of momentum $p_{c}$ at a very short-distance $\left(\sim 1 / p_{c_{T}} \sim 1 / Q_{T}\right)$, and (2) production of the lepton-pair via a virtual photon of invariant mass $Q$ through bremsstrahlung (or fragmentation) from the parton produced at the first stage. This two-stage production, shown in Fig. 司 or in general in Fig. 因, shares the same generic pattern of the fragmentation production of a single particle (e.g., a hadron of mass $M_{h}$ or a real photon) at large transverse momentum $Q_{T}$. If we neglect the power suppressed quantum interference between these two stages, the fragmentation (or bremsstrahlung) contributions should have the same general factored form that is present in single hadron or prompt photon production [12],

$$
\frac{d \hat{\sigma}_{a b \rightarrow \gamma^{*}(Q) X}^{(F)}}{d Q_{T}^{2} d y}=\sum_{c} \int \frac{d z}{z^{2}}\left[\frac{d \hat{\sigma}_{a b \rightarrow c X}^{(F)}}{d p_{c_{T}}^{2} d y}\left(x_{1}, x_{2}, p_{c}=\hat{Q} / z ; \mu_{F}\right)\right] D_{c \rightarrow \gamma^{*} X}\left(z, \mu_{F}^{2} ; Q^{2}\right) .
$$

Superscript $(F)$ indicates the fragmentation contribution; the sum $\sum_{c}$ runs over all parton flavors; and $\mu_{F}$ is the fragmentation scale defined below. The four vector $\hat{Q}^{\mu}$ in Eq. (8) is defined to be $Q^{\mu}$ but with $Q^{2}$ set to be zero.

The partonic cross sections, $d \hat{\sigma}_{a b \rightarrow c X} / d p_{c_{T}}^{2} d y$ in Eq. (8), represent the inclusive production of a parton of flavor $c$. These partonic cross sections are evaluated at a single hard scale $p_{c_{T}} \sim Q_{T}$. Exact perturbative expressions for the partonic cross sections depend on how the fragmentation functions $D_{c \rightarrow \gamma^{*} X}\left(z, \mu_{F}^{2} ; Q^{2}\right)$ are defined (or depend on the choices of the scheme) 12.

As for single hadron (or prompt photon) production, all large logarithms from the integration over the distance scale from $O\left(1 / p_{c_{T}}\right)$ (or $O\left(1 / Q_{T}\right)$ ) to $O(1 / Q)$, where the virtual photon is produced, are resummed into the fragmentation functions. The main difference for production of a virtual photon, a hadron, or a real photon is the difference in the fragmentation functions.

\section{B. Parton-to-Virtual-Photon Fragmentation Functions}

The fragmentation function for a parton of flavor $c$ into a virtual photon of invariant mass $\mathrm{Q}$ is defined as a probability density for finding the virtual photon with fraction $z$ of the parent parton's momentum. Because of the universality of fragmentation functions, virtual photon fragmentation functions can be derived in a process independent way [12] or extracted from a specific physical process. In this subsection, we derive the virtual photon fragmentation functions by resumming the leading logarithmic contributions to the DrellYan cross section at large transverse momentum. 
In order to resum the large logarithmic contributions to the Drell-Yan cross section to all orders and to extract the virtual photon fragmentation functions, we must identify all sources and the pattern of these logarithmic contributions. Consider a non-singlet fragmentation of a quark into a virtual photon, shown in Fig. 5. The virtual photon is produced from bremsstrahlung of a quark (or an antiquark). The quark (or antiquark) itself is produced either in the hard collision, as shown in Fig. 5(a), or from the fragmentation of another quark, as shown in Fig. 5(b) or Fig. 5(c). In a physical gauge (such as the light-cone gauge), the leading large logarithmic contributions of the subprocesses in Fig. 5 come from the part of phase space in which the daughter quark's invariant mass $k_{i}^{2}$ is much smaller than that of the parent quark $k_{i+1}^{2}$. In this situation, we can neglect all contributions suppressed by powers of $k_{i}^{2} / k_{i+1}^{2}$. The "decay" rate (or the fragmentation function) of the parent quark into a daughter quark plus massless partons is dominated by logarithmic contributions that are proportional to $\ln \left(k_{i+1}^{2} / k_{i}^{2}\right)$. The leading logarithmic contributions from the general fragmentation diagram in Fig. 4 arise from the region of strong ordering in the invariant masses of the fragmentation partons in Fig. 5: $p_{c}^{2} \gg \ldots k_{i+1}^{2} \gg k_{i}^{2} \gg \ldots \gg k_{0}^{2}$ [12].

With the strong ordering approximation, the leading logarithmic contributions from the non-singlet fragmentation in Fig. 5 can be factored into the hard production of a quark of momentum $p_{c}$ convoluted with a sum of all-orders fragmentation ladder diagrams, as shown in Fig. 6. By comparing the factored cross section in Eq. (8) with the factored expression in Fig. 6, and summing the ladder diagrams to all orders, one obtains the leading contributions to the non-singlet quark-to-virtual-photon fragmentation functions [12],

$$
\begin{aligned}
& D_{q \rightarrow \gamma^{*} X}^{N S}\left(z, p_{c}^{2} ; Q^{2}\right)= \int_{Q^{2} / z}^{p_{c}^{2}} \frac{d k_{0}^{2}}{k_{0}^{2}}\left(\frac{\alpha_{e m}}{2 \pi} \gamma_{q \rightarrow \gamma^{*}}^{(0)}\left(z, k_{0}^{2} ; Q^{2}\right)\right) \\
&+\sum_{n=1}^{\infty} {\left[\prod_{i=1}^{n} \int_{Q^{2} / z}^{k_{i+1}^{2}} \frac{d k_{i}^{2}}{k_{i}^{2}}\left(\frac{\alpha_{s}}{2 \pi} \int_{z_{i+1}}^{1} \frac{d z_{i}}{z_{i}} P_{q \rightarrow q}^{(0)}\left(\frac{z_{i+1}}{z_{i}}\right)\right)\right] } \\
& \times \int_{Q^{2} / z}^{k_{1}^{2}} \frac{d k_{0}^{2}}{k_{0}^{2}}\left(\frac{\alpha_{e m}}{2 \pi} \gamma_{q \rightarrow \gamma^{*}}^{(0)}\left(z_{1}, k_{0}^{2} ; Q^{2}\right)\right) .
\end{aligned}
$$

The superscript " $N S$ " represents the non-singlet contribution; the upper limit of integration is $k_{n+1}^{2}=p_{c}^{2}$; and the lower limit of integration, $Q^{2} / z$, is the mass threshold (or minimum invariant mass) for the quark to produce the virtual photon of invariant mass $Q$ [12]. The leading-order quark-to-quark splitting function $P_{q \rightarrow q}^{(0)}(z)$ is the same as the leadingorder quark-to-quark splitting function of the DGLAP evolution equations [16]. In deriving Eq. (9), we include the diagrams with quark wave function renormalization in addition to the ladder diagrams shown in Fig. 6. The function $\gamma_{q \rightarrow \gamma^{*}}^{(0)}\left(z, k_{0}^{2} ; Q^{2}\right)$ in Eq. (9) is the lowest order QED splitting function for a quark to fragment into a virtual photon [12, 17], defined below.

Since the invariant mass of the parent quark, $p_{c}^{2}$, can be very large in high energy collisions, the resummed logarithmic contributions, given by the second term on the right-handside of Eq. (9), can be important for the Drell-Yan cross section at low $Q^{2}$. From Eq. (8) and the fact that the partonic hard parts are evaluated at a single hard scale $\sim p_{c_{T}} \sim Q_{T}$, we conclude that all leading large logarithmic contributions at high $Q_{T}$ are included in the virtual photon fragmentation functions. Resummation of the large logarithmic contributions is equivalent to the derivation of the virtual photon fragmentation functions in Eq. (9). Unlike 
the parton-to-hadron fragmentation functions, the virtual photon fragmentation functions in Eq. (9) have no dependence on any non-perturbative momentum scale. The virtual photon's non-vanishing invariant mass removes the final-state collinear singularities that appear in the parton-to-hadron (or real photon) fragmentation functions. Therefore, all parton-tovirtual-photon fragmentation functions should be free of collinear singularities, and they are calculable in principle perturbatively to all orders in $\alpha_{s}$ [12].

By re-organizing the second term on the right-hand-side of Eq. (9), one may derive an integral equation for the non-singlet quark-to-virtual-photon fragmentation function,

$$
\begin{aligned}
D_{q \rightarrow \gamma^{*} X}^{N S}\left(z, p_{c}^{2} ; Q^{2}\right) & =\int_{Q^{2} / z}^{p_{c}^{2}} \frac{d k_{0}^{2}}{k_{0}^{2}}\left(\frac{\alpha_{e m}}{2 \pi} \gamma_{q \rightarrow \gamma^{*}}^{(0)}\left(z, k_{0}^{2} ; Q^{2}\right)\right) \\
& +\int_{Q^{2} / z}^{p_{c}^{2}} \frac{d k^{2}}{k^{2}}\left(\frac{\alpha_{s}}{2 \pi} \int_{z}^{1} \frac{d z^{\prime}}{z^{\prime}} P_{q \rightarrow q}^{(0)}\left(\frac{z}{z^{\prime}}\right)\right) D_{q \rightarrow \gamma^{*} X}^{N S}\left(z^{\prime}, k^{2} ; Q\right) .
\end{aligned}
$$

Carrying out the sum over all-orders logarithmic contributions in Eq. (9) is equivalent to solving the integral equation in Eq. (10).

We introduce fragmentation scale $\mu_{F}$ and let $\mu_{F}^{2} \equiv p_{c}^{2}$, the square of the invariant mass of the parent quark in Eq. (10). One can derive an evolution equation by applying $\mu_{F}^{2} d / d \mu_{F}^{2}$ to both sides of Eq. (10),

$$
\begin{aligned}
\mu_{F}^{2} \frac{d}{d \mu_{F}^{2}} D_{q \rightarrow \gamma^{*} X}^{N S}\left(z, \mu_{F}^{2} ; Q^{2}\right) & =\frac{\alpha_{e m}}{2 \pi} \gamma_{q \rightarrow \gamma^{*}}^{(0)}\left(z, \mu_{F}^{2} ; Q^{2}\right) \\
& +\frac{\alpha_{s}}{2 \pi} \int_{z}^{1} \frac{d z^{\prime}}{z^{\prime}} P_{q \rightarrow q}^{(0)}\left(\frac{z}{z^{\prime}}\right) D_{q \rightarrow \gamma^{*} X}^{N S}\left(z^{\prime}, \mu_{F}^{2} ; Q^{2}\right) .
\end{aligned}
$$

Because the quark can interact directly with the virtual photon, the evolution equation in Eq. (11) has an inhomogeneous term.

By extending the simple ladder diagrams in Fig. 6 to general ladder diagrams [18, one can derive evolution equations for the singlet quark-to-virtual-photon and gluon-to-virtualphoton fragmentation functions [12],

$$
\begin{aligned}
\mu_{F}^{2} \frac{d}{d \mu_{F}^{2}} D_{c \rightarrow \gamma^{*} X}\left(z, \mu_{F}^{2} ; Q^{2}\right) & =\left(\frac{\alpha_{e m}}{2 \pi}\right) \gamma_{c \rightarrow \gamma^{*}}\left(z, \mu_{F}^{2}, \alpha_{s} ; Q^{2}\right) \\
& +\left(\frac{\alpha_{s}}{2 \pi}\right) \sum_{d} \int_{z}^{1} \frac{d z^{\prime}}{z^{\prime}} P_{c \rightarrow d}\left(\frac{z}{z^{\prime}}, \alpha_{s}\right) D_{d \rightarrow \gamma^{*} X}\left(z^{\prime}, \mu_{F}^{2} ; Q^{2}\right),
\end{aligned}
$$

where $c, d=q, \bar{q}, g$. In Eq. (12), the evolution kernels $P_{c \rightarrow d}$ are evaluated at a single hard scale, $\mu_{F}$, and can be calculated perturbatively as a power series in $\alpha_{s}$. QCD corrections to the QED quark-to-virtual-photon splitting function $\gamma_{c \rightarrow \gamma^{*}}$ can be evaluated in principle order-by-order in $\alpha_{s}$.

Calculating the lowest order quark-to-virtual-photon ladder diagram in Fig. 6, we obtain the leading order quark-to-virtual-photon QED evolution kernel [12, 17],

$$
\gamma_{q \rightarrow \gamma^{*}}^{(0)}\left(z, k^{2} ; Q^{2}\right)=e_{q}^{2}\left[\frac{1+(1-z)^{2}}{z}-z\left(\frac{Q^{2}}{z k^{2}}\right)\right] \theta\left(k^{2}-\frac{Q^{2}}{z}\right) .
$$

The $\theta$-function is a consequence of the mass threshold. The gluon-to-virtual-photon evolution kernel vanishes at the same order 


$$
\gamma_{g \rightarrow \gamma^{*}}^{(0)}\left(z, k^{2} ; Q^{2}\right)=0
$$

because the gluon does not interact directly with the virtual photon.

It is important to note that if we work in the strict leading power (or leading twist) approximation, we would drop both the power corrections to the fragmentation functions as well as power corrections to the evolution kernels of the fragmentation functions. That is, we would neglect the $O\left(Q^{2} / k^{2}\right)$ term in Eq. (13),

$$
\gamma_{q \rightarrow \gamma^{*}}^{(L P-0)}\left(z, k^{2}\right)=\gamma_{q \rightarrow \gamma^{*}}^{(0)}\left(z, k^{2} ; Q^{2}=0\right)=e_{q}^{2}\left[\frac{1+(1-z)^{2}}{z}\right] \theta\left(k^{2}-\frac{Q^{2}}{z}\right) .
$$

The superscript "LP" represents the leading power approximation. With this strictly leading power quark-to-photon QED evolution kernel, the evolution equation in Eq. (12) for the parton-to-virtual-photon fragmentation functions is exactly the same as that for the partonto-real-photon fragmentation functions [13]. Under the strict leading power approximation, the only difference between virtual and real photon fragmentation functions is the boundary condition for the evolution equations. For real photon fragmentation functions, a set of unknown non-perturbative input fragmentation functions $D_{c \rightarrow \gamma X}(z)$ is needed at a given scale $\mu_{F}^{0}(\sim$ a few $\mathrm{GeV})$ [19]. On the other hand, no non-perturbative input fragmentation functions are needed to solve the evolution equations in Eq. (12). Instead, the mass threshold for production of a time-like virtual photon of invariant mass $Q$ imposes a natural boundary condition for all flavors $c$,

$$
D_{c \rightarrow \gamma^{*} X}\left(z, \mu_{F}^{2} \leq Q^{2} / z ; Q^{2}\right)=0 .
$$

The strict leading-power approximation might be too severe in the threshold region [12]. Because its mass is non-zero, the virtual photon can have both transverse and longitudinal polarization modes. The QED evolution kernel in Eq. (13) is a sum of evolution kernels for a quark to fragment into either transverse $(T)$ or longitudinal $(L)$ polarization modes [12, 17,

$$
\gamma_{q \rightarrow \gamma^{*}}^{(0)}\left(z, k^{2} ; Q^{2}\right)=2 \gamma_{q \rightarrow \gamma_{T}^{*}}^{(0)}\left(z, k^{2} ; Q^{2}\right)+\gamma_{q \rightarrow \gamma_{L}^{*}}^{(0)}\left(z, k^{2} ; Q^{2}\right)
$$

with

$$
\begin{aligned}
& \gamma_{q \rightarrow \gamma_{T}^{*}}^{(0)}\left(z, k^{2} ; Q^{2}\right)=e_{q}^{2} \frac{1}{2}\left(\frac{1+(1-z)^{2}}{z}\right)\left[1-\frac{Q^{2}}{z k^{2}}\right] \theta\left(k^{2}-\frac{Q^{2}}{z}\right) ; \\
& \gamma_{q \rightarrow \gamma_{L}^{*}}^{(0)}\left(z, k^{2} ; Q^{2}\right)=e_{q}^{2}\left[2\left(\frac{1-z}{z}\right)\right]\left(\frac{Q^{2}}{z k^{2}}\right) \theta\left(k^{2}-\frac{Q^{2}}{z}\right) .
\end{aligned}
$$

The factor 2 in Eq. (17) represents the two transverse polarization states of the virtual photon. Under the strict leading power approximation, Eq. (19) vanishes. In this strict limit, only transversely polarized virtual photons are produced through the fragmentation processes, and there are no logarithmic contributions to the production of longitudinally polarized virtual photons. Furthermore, without the $O\left(Q^{2} / k^{2}\right)$ term in Eq. (18), the evolution kernel $\gamma_{q \rightarrow \gamma_{T}^{*}}^{(0)}\left(z, k^{2} ; Q^{2}\right)$ gives the wrong threshold behavior. Instead of being zero at the threshold when $k^{2}=Q^{2} / z$, the leading power evolution kernel $\gamma_{q \rightarrow \gamma_{T}^{*}}^{(L P-0)}\left(z, k^{2} ; Q^{2}\right)$ is finite and proportional to $1 / z$. It is large if $z$ is small. 
In general, there can be two types of power corrections to the leading power virtual photon fragmentation functions. Power suppressed contributions $O\left(Q^{2} / \mu_{F}^{2}\right)$ to the fragmentation functions are one type (Type-I as defined in Ref. [11]), and power corrections contributions to the evolution kernels (or the slopes) of the fragmentation functions are the other type (known as Type-II). For example, consider the lowest order contribution to the quark-to-virtual-photon fragmentation function from the evolution kernel in Eq. (18),

$$
\begin{aligned}
D_{q \rightarrow \gamma_{T}^{*} X}^{(0)}\left(z, \mu_{F}^{2} ; Q^{2}\right) & \equiv \int_{Q^{2} / z}^{\mu_{F}^{2}} \frac{d k^{2}}{k^{2}}\left(\frac{\alpha_{e m}}{2 \pi}\right) \gamma_{q \rightarrow \gamma_{T}^{*}}^{(0)}\left(z, k^{2} ; Q^{2}\right) \\
& =e_{q}^{2}\left(\frac{\alpha_{e m}}{2 \pi}\right) \frac{1}{2}\left(\frac{1+(1-z)^{2}}{z}\right)\left[\ln \left(\frac{z \mu_{F}^{2}}{Q^{2}}\right)-\left(1-\frac{Q^{2}}{z \mu_{F}^{2}}\right)\right] .
\end{aligned}
$$

The $\left(1-Q^{2} / z \mu_{F}^{2}\right)$ term results from the power suppressed $Q^{2} / z k^{2}$ term in the evolution kernel, and it is clear that this term is as important as the logarithmic term in the threshold region. The term proportional to 1 in this $\left(1-Q^{2} / z \mu_{F}^{2}\right)$ combination is not power suppressed by $O\left(Q^{2} / \mu_{F}^{2}\right)$. In the threshold region, the virtual photon fragmentation functions are dominated by the longitudinally polarized component particularly when $z$ is small [12]. The Type-II power corrections are not necessarily small and could provide power non-suppressed contributions to physical observables [11].

In the rest of our discussion, we keep the leading power suppressed terms in the QED evolution kernels in Eq. (13) when we calculate our parton-to-virtual-photon fragmentation functions. As shown in Ref. [12], the inhomogeneous QED evolution kernels in Eq. (12) dominate the scale dependence of the fragmentation functions, and therefore, we neglect the power corrections to the QCD evolution kernels $P_{c \rightarrow d}$ in Eq. (12). With the inclusion of power corrections in the evolution kernels, the resummation discussed here is no longer a simple one-scale problem in QCD perturbation theory. More detailed discussions of the virtual-photon fragmentation functions can be found in Ref. [12].

In summary, the all-orders resummation of the large logarithmic contributions to the low mass Drell-Yan cross section is equivalent to a sum of all logarithmic contributions to the virtual photon fragmentation functions, achieved by solution of the evolution equations in Eq. (12). The evolution equations for the virtual photon fragmentation functions have the same functional forms as those for real photon fragmentation functions [13]. However, the differences in both boundary conditions and the inhomogeneous terms due to the nonvanishing of $Q^{2}$ lead to many differences between the real and virtual photon fragmentation functions. One major difference is that the virtual photon fragmentation functions are purely perturbative. In addition, the virtual photon fragmentation functions provide the resummed contributions to the production of a longitudinally polarized virtual photon [12].

\section{Calculation of the Partonic Hard Parts}

To determine the fragmentation contributions to the Drell-Yan cross section,

$$
\frac{d \sigma_{A B \rightarrow \gamma^{*}(Q) X}^{(F)}}{d Q_{T}^{2} d y}=\sum_{a, b} \int d x_{1} \phi_{a / A}\left(x_{1}, \mu\right) \int d x_{2} \phi_{b / B}\left(x_{2}, \mu\right)
$$




$$
\times \sum_{c} \int \frac{d z}{z^{2}}\left[\frac{d \hat{\sigma}_{a b \rightarrow c X}^{(F)}}{d p_{c_{T}}^{2} d y}\left(x_{1}, x_{2}, p_{c}=\hat{Q} / z ; \mu_{F}\right)\right] D_{c \rightarrow \gamma^{*} X}\left(z, \mu_{F}^{2} ; Q^{2}\right)
$$

we must evaluate the partonic hard parts $d \hat{\sigma}_{a b \rightarrow c X}^{(F)} / d p_{c_{T}}^{2} d y$ in Eq. (21). Although these are calculable perturbatively, exact expressions depend on how the parton distributions and fragmentation functions are defined (or the choice of factorization scheme). In this subsection, we provide a self-consistent procedure for the calculation of the partonic hard parts.

We separate the procedure into four steps: (1) instead of considering the hadronic process: $A+B \rightarrow \gamma^{*}(Q) X$, we apply the factored formula in Eq. (21) to a partonic process: $a^{\prime}+b^{\prime} \rightarrow c^{\prime} X$ with an on-shell final-state parton $c^{\prime}$; (2) we expand both sides of the factored formula for the partonic process order-by-order in $\alpha_{s}$; (3) we calculate the partonic cross section $d \sigma_{a^{\prime} b^{\prime} \rightarrow c^{\prime} X} / d p_{c_{T}^{\prime}}^{2} d y$, and parton-to-parton distributions and fragmentation functions order-by-order in $\alpha_{s}$; and (4) we extract the short-distance partonic hard parts $d \hat{\sigma}_{a b \rightarrow c X} / d p_{c_{T}}^{2} d y$ by comparing both sides of the perturbatively expanded factored formula at the same order of $\alpha_{s}$.

By applying the fragmentation expression in Eq. (21) to the partonic processes: $a^{\prime}+b^{\prime} \rightarrow$ $c^{\prime} X$, we obtain the following schematic formula

$$
\sigma_{a^{\prime} b^{\prime} \rightarrow c^{\prime}}=\sum_{a, b, c} \phi_{a / a^{\prime}} \otimes \phi_{b / b^{\prime}} \otimes \hat{\sigma}_{a b \rightarrow c}^{(F)} \otimes D_{c \rightarrow c^{\prime}}
$$

Symbol $\otimes$ represents the convolutions over momentum fractions $x_{1}, x_{2}$, and $z$ in Eq. (21). (In Eq. (22) and in the rest of the equations of this subsection, we omit the inclusive symbol $X$ for reasons of notational simplicity.)

To produce a parton with large transverse momentum, a $2 \rightarrow 2$ partonic process is required at the parton level, at minimum of the order $O\left(\alpha_{s}^{2}\right)$. Expanding both sides of the factored formula Eq. (22) order-by-order in $\alpha_{s}$, we define the following perturbative expansions,

$$
\begin{aligned}
\sigma_{a^{\prime} b^{\prime} \rightarrow c^{\prime}} & \equiv \sum_{n=2} \sigma_{a^{\prime} b^{\prime} \rightarrow c^{\prime}}^{(n)}\left(\frac{\alpha_{s}(\mu)}{2 \pi}\right)^{n}, \\
\hat{\sigma}_{a b \rightarrow c}^{(F)} & \equiv \sum_{n=2} H_{a b \rightarrow c}^{(n)}\left(\frac{\alpha_{s}(\mu)}{2 \pi}\right)^{n}, \\
\phi_{a / a^{\prime}} & \equiv \sum_{n=0} \phi_{a / a^{\prime}}^{(n)}\left(\frac{\alpha_{s}(\mu)}{2 \pi}\right)^{n}, \\
D_{c \rightarrow c^{\prime}} & \equiv \sum_{n=0} D_{c \rightarrow c^{\prime}}^{(n)}\left(\frac{\alpha_{s}(\mu)}{2 \pi}\right)^{n} .
\end{aligned}
$$

We substitute these four perturbative expansions into Eq. (22) and obtain at $O\left(\alpha_{s}^{2}\right)$,

$$
\sigma_{a^{\prime} b^{\prime} \rightarrow c^{\prime}}^{(2)}=\sum_{a, b, c} \phi_{a / a^{\prime}}^{(0)} \otimes \phi_{b / b^{\prime}}^{(0)} \otimes H_{a b \rightarrow c}^{(2)} \otimes D_{c \rightarrow c^{\prime}}^{(0)} .
$$

Since the zeroth order parton distributions and fragmentation functions are $\delta$-functions, Eq. (27) yields $H_{a b \rightarrow c}^{(2)}=\sigma_{a b \rightarrow c}^{(2)}$ or 


$$
\frac{d \hat{\sigma}_{a b \rightarrow c}^{(F-L O)}}{d p_{c_{T}}^{2} d y}=\frac{d \sigma_{a b \rightarrow c}^{(L O)}}{d p_{c_{T}}^{2} d y}
$$

at leading order.

Expanding Eq. (22) to NLO, we write

$$
\begin{aligned}
\sigma_{a^{\prime} b^{\prime} \rightarrow c^{\prime}}^{(3)} & =\sum_{a, b, c} \phi_{a / a^{\prime}}^{(0)} \otimes \phi_{b / b^{\prime}}^{(0)} \otimes H_{a b \rightarrow c}^{(3)} \otimes D_{c \rightarrow c^{\prime}}^{(0)} \\
& +\sum_{a, b, c} \phi_{a / a^{\prime}}^{(1)} \otimes \phi_{b / b^{\prime}}^{(0)} \otimes H_{a b \rightarrow c}^{(2)} \otimes D_{c \rightarrow c^{\prime}}^{(0)} \\
& +\sum_{a, b, c} \phi_{a / a^{\prime}}^{(0)} \otimes \phi_{b / b^{\prime}}^{(1)} \otimes H_{a b \rightarrow c}^{(2)} \otimes D_{c \rightarrow c^{\prime}}^{(0)} \\
& +\sum_{a, b, c} \phi_{a / a^{\prime}}^{(0)} \otimes \phi_{b / b^{\prime}}^{(0)} \otimes H_{a b \rightarrow c}^{(2)} \otimes D_{c \rightarrow c^{\prime}}^{(1)} .
\end{aligned}
$$

Using the zero-th order parton distributions and fragmentation functions, for $d \hat{\sigma}_{a b \rightarrow c}^{(F-N L O)} / d p_{c_{T}}^{2} d y$, we obtain

$$
\begin{aligned}
H_{a b \rightarrow c}^{(3)} & =\sigma_{a b \rightarrow c}^{(3)}-\sum_{a^{\prime}} \phi_{a^{\prime} / a}^{(1)} \otimes H_{a^{\prime} b \rightarrow c}^{(2)} \\
& -\sum_{b^{\prime}} \phi_{b^{\prime} / b}^{(1)} \otimes H_{a b^{\prime} \rightarrow c}^{(2)}-\sum_{c^{\prime}} H_{a b \rightarrow c^{\prime}}^{(2)} \otimes D_{c^{\prime} \rightarrow c^{\prime}}^{(1)} .
\end{aligned}
$$

In Eq. (30), $H_{a b \rightarrow c}^{(2)}$ is the leading order contribution calculated in Eq. (28). The partonic cross section $\sigma_{a b \rightarrow c}^{(3)}$ and all parton-level parton distributions and fragmentation functions are perturbatively calculable with proper regulators. The subtraction terms in Eq. (30) remove the collinear singularities associated with the massless partons. Following the same procedure, we can derive the short-distance partonic hard parts for the fragmentation contributions in Eq. (21) at all orders in $\alpha_{s}$.

Equation (30) shows that beyond the leading order, the exact expressions for the perturbatively calculated short-distance hard parts depend on the definitions of parton-level parton distributions and fragmentation functions. The partonic hard parts are fixed uniquely once we fix the parton-level parton distributions and fragmentation functions. In order to use available conventional parton distributions, we have little choice other than to select the parton-level parton distributions in either the $\overline{M S}$ or DIS scheme 20].

In the partonic cross section $\sigma_{a b \rightarrow c}^{(n)}$, the parton momentum $p_{c}$ is assumed to be massless, $p_{c}^{2}=0$, and, therefore, a final-state collinear singularity arises. Within the usual QCD factorization framework, there is freedom to choose any factorization scheme to remove the final-state collinear singularities of the partonic cross section and absorb all possible finite differences into the non-perturbative fragmentation functions. Different choices for the factorization scheme lead to finite differences between the extracted non-perturbative fragmentation functions. However, owing to the non-zero invariant mass of the virtual photon, the parton-to-virtual-photon fragmentation functions do not have final-state collinear singularities. They are completely perturbative. Therefore, the parton-to-virtual-photon fragmentation functions cannot uniquely fix the definition of the parton-to-parton fragmentation functions. As a consequence of the difference in the invariant masses of the parton 
and the virtual photon, an extra constraint has to be introduced to specify the parton-toparton fragmentation functions $D_{c^{\prime} \rightarrow c}^{(n)}$. We can choose a scheme for defining the parton-level fragmentation functions so as to remove the final-state collinear singularities in the partonic cross sections $\sigma_{a b \rightarrow c}^{(n)}$, with $n \geq 3$. The finite differences between schemes cannot be completely absorbed into the perturbative parton-to-virtual-photon fragmentation functions.

Finite differences associated with the choice of scheme for the parton-level fragmentation functions correspond to non-logarithmic contributions to the Drell-Yan cross section. For the logarithmic contributions to the Drell-Yan fragmentation functions, we can choose partonlevel fragmentation functions in the $\overline{M S}$ scheme or in any other scheme when calculating the partonic short-distance hard parts in Eq. (29). The perturbatively calculated partonic hard parts $d \hat{\sigma}_{a b \rightarrow c}^{(F)} / d p_{c_{T}}^{2} d y$ will be the same as those for inclusive single hadron (or prompt photon) production if the same factorization scheme is used. The non-logarithmic differences caused by the different choices of the factorization schemes can be absorbed into the direct contributions to the Drell-Yan cross section, defined and discussed in next section.

\section{DRELL-YAN CROSS SECTION WITH RESUMMED FRAGMENTATION CONTRIBUTIONS}

In this section, we derive our modified factorization formula for the Drell-Yan cross section including resummation of the logarithmic contributions.

Particularly at leading order, but also at all higher orders in perturbation theory, there are significant contributions to the cross section that are not included in the fragmentation terms. It is essential that the full factorization formula include all non-logarithmic contributions order-by-order in $\alpha_{s}$, while retaining the all orders resummation of the logarithmic contributions.

We use the word direct to designate the non-logarithmic contributions to the Drell-Yan cross section. This use of the term is the same as its use in prompt photon production. At leading order the direct terms in the Drell Yan cross section are those supplied by the shortdistance $O\left(1 / Q_{T}\right)$ 2-parton to 2-parton Compton and annihilation subprocesses. However, there is an additional component at higher orders. The direct contribution must also absorb the finite non-logarithmic differences in the partonic hard parts $H_{a b \rightarrow c X}^{(m)}$ associated with the ambiguity in definition of the parton-level fragmentation functions, as discussed in the last section. This second component accounts for the non-logarithmic terms over the distance interval $O(1 / Q)$ to $O\left(1 / Q_{T}\right)$. The logarithmic terms are included in the fragmentation functions. The physics of our direct term is very similar to that represented by the $Y$ term in the CSS formalism [9] for resummation at small $Q_{T}$.

To be precise, we define the direct contribution as the difference

$$
\begin{aligned}
\frac{d \sigma_{A B \rightarrow \gamma^{*}(Q) X}^{(D i r)}}{d Q_{T}^{2} d y} & \equiv \frac{d \sigma_{A B \rightarrow \gamma^{*}(Q) X}}{d Q_{T}^{2} d y}-\frac{d \sigma_{A B \rightarrow \gamma^{*}(Q) X}^{(F)}}{d Q_{T}^{2} d y} \\
& =\sum_{a, b} \int d x_{1} \phi_{a / A}\left(x_{1}, \mu\right) \int d x_{2} \phi_{b / B}\left(x_{2}, \mu\right)\left[\frac{d \hat{\sigma}_{a b \rightarrow \gamma^{*}(Q) X}^{(D i r)}}{d Q_{T}^{2} d y}\right] .
\end{aligned}
$$

The parton level direct term is obtained from the factored formulas in Eqs. (2) and (21), 


$$
\begin{aligned}
\frac{d \hat{\sigma}_{a b \rightarrow \gamma^{*}(Q) X}^{(D i r)}}{d Q_{T}^{2} d y}\left(x_{1}, x_{2}, Q, Q_{T}, y ; \mu, \mu_{F}\right) & \equiv \frac{d \hat{\sigma}_{a b \rightarrow \gamma^{*}(Q) X}}{d Q_{T}^{2} d y}\left(x_{1}, x_{2}, Q, Q_{T}, y ; \mu\right) \\
& -\frac{d \hat{\sigma}_{a b \rightarrow \gamma^{*}(Q) X}^{(F)}}{d Q_{T}^{2} d y}\left(x_{1}, x_{2}, Q, Q_{T}, y ; \mu, \mu_{F}\right)
\end{aligned}
$$

with $d \hat{\sigma}_{a b \rightarrow \gamma^{*}(Q) X}^{(F)} / d Q_{T}^{2} d y$ given in Eq. (8) and $d \hat{\sigma}_{a b \rightarrow \gamma^{*}(Q) X} / d Q_{T}^{2} d y$ calculated in conventional fixed-order QCD perturbation theory. Since $d \hat{\sigma}_{a b \rightarrow \gamma^{*}(Q) X} / d Q_{T}^{2} d y$ and $d \hat{\sigma}_{a b \rightarrow \gamma^{*}(Q) X}^{(F)} / d Q_{T}^{2} d y$ are calculable perturbatively, the direct contributions, $d \hat{\sigma}_{a b \rightarrow \gamma^{*}(Q) X}^{(D i r)} / d Q_{T}^{2} d y$ in Eq. (32), should also be calculable perturbatively. They have the perturbative expansion

$$
\frac{d \hat{\sigma}_{a b \rightarrow \gamma^{*}(Q) X}^{(D i r)}}{d Q_{T}^{2} d y}=\sum_{n=1} Y_{a b \rightarrow \gamma^{*}(Q) X}^{(n)}\left(x_{1}, x_{2}, Q, Q_{T}, y ; \mu_{F}, \mu\right)\left(\frac{\alpha_{s}(\mu)}{2 \pi}\right)^{n},
$$

where $n \geq 1$ because the leading order contributions to Drell-Yan cross section are of order $\bar{O}\left(\alpha_{e m} \alpha_{s}\right)$. Since the fragmentation contributions $d \hat{\sigma}_{a b \rightarrow \gamma^{*}(Q) X}^{(F)} / d Q_{T}^{2} d y$ and $d \hat{\sigma}_{a b \rightarrow \gamma^{*}(Q) X} / d Q_{T}^{2} d y$ share the same large logarithmic terms, $\ln ^{m}\left(Q_{T}^{2} / Q^{2}\right)$, the direct contributions should be free of large logarithms order-by-order in $\alpha_{s}$. Therefore, the coefficient functions $Y_{a b \rightarrow \gamma^{*}(Q) X}^{(n)}$ in Eq. (33) are evaluated at a single hard scale $\left(\sim Q_{T}\right)$, and the perturbative expansion for the direct contribution in Eq. (33) is expected to be well-behaved perturbatively. We substitute Eqs. (3), (24), (26), and (33) into Eq. (32) and obtain

$$
Y_{a b \rightarrow \gamma^{*}(Q) X}^{(n)}=H_{a b \rightarrow \gamma^{*}(Q) X}^{(n)}-\sum_{m=2}^{n}\left[\sum_{c} H_{a b \rightarrow c X}^{(m)} \otimes D_{c \rightarrow \gamma^{*}(Q) X}^{(n-m)}\right] .
$$

Functions $D_{c \rightarrow \gamma^{*}(Q) X}^{(n-m)}$ are the coefficient functions for the perturbatively calculated parton-tovirtual-photon fragmentation functions [12]. The subtraction term in Eq. (34) removes the logarithmic contributions included in the coefficient functions $H_{a b \rightarrow \gamma^{*}(Q) X}$ that are calculated in conventional fixed-order perturbation theory. As remarked above, the direct contributions defined in Eq. (34) absorb the finite non-logarithmic differences in the partonic hard parts $H_{a b \rightarrow c X}^{(m)}$. If $H_{a b \rightarrow c X}^{(m)}$ is larger in one scheme and more is included in the fragmentation contributions, then more will be subtracted from the direct contributions. Any non-compensated differences will be higher order corrections in $\alpha_{s}$.

We substitute Eq. (32) into Eq. (31) and derive a modified factorization formula for the Drell-Yan cross section at $Q_{T} \geq Q$ :

$$
\begin{aligned}
\frac{d \sigma_{A B \rightarrow \gamma^{*}(Q) X}}{d Q_{T}^{2} d y}= & \sum_{a, b} \int d x_{1} \phi_{a / A}\left(x_{1}, \mu\right) \int d x_{2} \phi_{b / B}\left(x_{2}, \mu\right) \\
& \times\left\{\frac{d \hat{\sigma}_{a b \rightarrow \gamma^{*}(Q) X}^{(D i r)}}{d Q_{T}^{2} d y}\left(x_{1}, x_{2}, Q, Q_{T}, y ; \mu_{F}, \mu\right)\right. \\
& \left.+\sum_{c} \int \frac{d z}{z^{2}}\left[\frac{d \hat{\sigma}_{a b \rightarrow c X}^{(F)}}{d p_{c_{T}}^{2} d y}\left(x_{1}, x_{2}, p_{c}=\frac{\hat{Q}}{z} ; \mu_{F}, \mu\right)\right] D_{c \rightarrow \gamma^{*}(Q) X}\left(z, \mu_{F}^{2} ; Q^{2}\right)\right\} .
\end{aligned}
$$

Both perturbatively calculated short-distance hard parts, $d \hat{\sigma}_{a b \rightarrow c X}^{(F)} / d p_{c_{T}}^{2} d y$ and $d \hat{\sigma}_{a b \rightarrow \gamma^{*}(Q) X}^{(D i r)} / d Q_{T}^{2} d y$, are free of large logarithms. All potentially large $\ln ^{m}\left(Q_{T}^{2} / Q^{2}\right)$ 
terms in the region $Q_{T}^{2} \gg Q^{2}$ are resummed into the virtual photon fragmentation functions $D_{c \rightarrow \gamma^{*}(Q) X}$. The direct contribution (first term on the right-hand side of Eq. (35)) represents the production of lepton-pairs at the distance scale of the hard collisions $\left(\sim 1 / Q_{T}\right)$. The fragmentation contribution stands for the sum of all leading logarithmic contributions from a distance scale at $1 / Q_{T}$ to $1 / Q$.

We note here, in passing, that unlike the logarithmic terms in the region of small $Q_{T}$, the logarithmic terms at large $Q_{T}$ are typical DGLAP logarithms associated with collinear contributions. At low $Q_{T}$, logarithmic contributions arise from the collinear and the infrared regions. Correspondingly, in resummation formalisms relevant at small $Q_{T}$, there are leading log terms (including both collinear and infrared logs at all orders in $\alpha_{s}$ ), and next-to-leading log terms (including only one of the two types of logs), and "next-to-next-to-leading" log terms, and so forth. In our case, we do not have the type of "next-to-leading" logs that appear in resummation procedures at small $Q_{T}$. Anything left-over in the large $Q_{T}$ region, after resummation of the leading logs of DGLAP type, is included in the direct terms.

Since we have in mind applications at large $Q_{T}$ but small $Q$, it is important to consider the possible role of higher-twist contributions proportional to inverse-powers of $Q$. Like power corrections from target mass effects, power corrections in our case should appear in the form of $m^{2} /$ (energy exchange $)^{2}$, with $m \sim Q$. Since $Q_{T}^{2}$ sets the hard scale, and $Q^{2}$ acts as a mass threshold, the only dimensionless ratios we expect to see are $Q^{2} / Q_{T}^{2}$ and $Q^{2} / \mu^{2}$, but no $1 / Q^{2}$ term. There can be three kinds of power corrections: power corrections to the partonic hard parts, suppressed by $Q^{2} / Q_{T}^{2}$; power corrections to the fragmentation contributions, suppressed by $Q^{2} / \mu_{F}^{2} \sim Q^{2} / Q_{T}^{2}$; and power corrections to the evolution kernels of the fragmentation functions, proportional to $Q^{2} / \mu^{2}$. Since $\mu^{2}$ runs from $Q^{2}$ to $\mu_{F}^{2}$, the third type could be significant, $Q^{2} / \mu_{\min }^{2} \sim O(1)$. Although this third variety is potentially important, the terms should be much smaller than the leading logarithmic contributions as long as $\ln \left(Q_{T}^{2} / Q^{2}\right) \gg 1$.

Our modified factorization formula for the Drell-Yan cross section in Eq. (35) is very similar to that for prompt real photon production in Eq. (5). However, the $Q^{2}$-dependence in the direct production term and differences in the fragmentation functions distinguish the Drell-Yan virtual photon production from prompt real photon production.

The key difference between our modified factorization formula in Eq. (35) and the conventional factorization formula in Eq. (2) resides in the way the logarithmic contributions from final-state parton splitting are handled. Instead of one perturbative series in powers of $\alpha_{s}$ in the conventional approach, we have two perturbative expansions in our modified factorization formula: one for the direct and one for the fragmentation contribution. All coefficient functions in the new perturbative expansions are free of large logarithms. The large logarithms in the conventional perturbative expansion are resummed into the fragmentation functions. In the rest of this section, we demonstrate the difference by comparing the two factorization formulas explicitly order-by-order in powers of $\alpha_{s}$.

According to the conventional factorization approach, the lowest order partonic contributions to virtual photon production are provided by the quark-gluon Compton and quark-antiquark annihilation diagrams at order $\alpha_{e m} \alpha_{s}$ : 


$$
\frac{d \sigma_{A B \rightarrow \gamma^{*}(Q) X}^{(C-L O)}}{d Q_{T}^{2} d y}=\sum_{a, b} \int d x_{1} \phi_{a / A}\left(x_{1}, \mu\right) \int d x_{2} \phi_{b / B}\left(x_{2}, \mu\right)\left[\frac{d \hat{\sigma}_{a b \rightarrow \gamma^{*}(Q) X}^{(C-L O)}}{d Q_{T}^{2} d y}\left(x_{1}, x_{2}, Q, Q_{T}, y ; \mu\right)\right]
$$

The superscript $(C-L O)$ stands for the leading order conventional perturbative calculation.

In our modified factorization approach, Eq. (35), there are two perturbative expansions corresponding to the direct and fragmentation contributions, respectively. Each perturbation series has its own leading order contributions. Because the subtraction term in Eq. (34) starts at $O\left(\alpha_{s}^{2}\right)$, the LO direct contribution is the same as the LO conventional contribution in Eq. (36),

$$
\frac{d \sigma_{A B \rightarrow \gamma^{*}(Q) X}^{(D i r-L O)}}{d Q_{T}^{2} d y}=\sum_{a, b} \int d x_{1} \phi_{a / A}\left(x_{1}, \mu\right) \int d x_{2} \phi_{b / B}\left(x_{2}, \mu\right)\left[\frac{d \hat{\sigma}_{a b \rightarrow \gamma^{*}(Q) X}^{(D i r-L O)}}{d Q_{T}^{2} d y}\left(x_{1}, x_{2}, Q, Q_{T}, y ; \mu\right)\right] .
$$

The LO partonic hard part $d \hat{\sigma}_{a b \rightarrow \gamma^{*}(Q) X}^{(D i r-L O)} / d Q_{T}^{2} d y$ equals $d \hat{\sigma}_{a b \rightarrow \gamma^{*}(Q) X}^{(C-L O)} / d Q_{T}^{2} d y$ in Eq. (36).

The virtual photon fragmentation functions are of order $\alpha_{e m}$ [12], and the partonic hard parts, $d \hat{\sigma}_{a b \rightarrow c X}^{(F)} / d p_{c_{T}}^{2} d y$ in Eq. (35) start at order $\alpha_{s}^{2}$. The LO fragmentation contributions to the Drell-Yan cross section would seem therefore to be of order $O\left(\alpha_{e m} \alpha_{s}^{2}\right)$, one power of $\alpha_{s}$ higher than the LO direct contributions in Eq. (37). However, the large logarithms from the virtual photon fragmentation functions are proportional to $\ln \left(\mu_{F}^{2}\right) \propto 1 / \alpha_{s}\left(\mu_{F}\right)$, meaning that the LO fragmentation contributions to the Drell-Yan cross section can be viewed as effectively $O\left(\alpha_{e m} \alpha_{s}\right)$ terms, of the same order as the LO direct contribution:

$$
\begin{aligned}
\frac{d \sigma_{A B \rightarrow \gamma^{*}(Q) X}^{(F-L O)}}{d Q_{T}^{2} d y}= & \sum_{a, b} \int d x_{1} \phi_{a / A}\left(x_{1}, \mu\right) \int d x_{2} \phi_{b / B}\left(x_{2}, \mu\right) \\
& \times \sum_{c} \int \frac{d z}{z^{2}} \frac{d \hat{\sigma}_{a b \rightarrow c X}^{(F-L O)}}{d p_{c_{T}}^{2} d y}\left(x_{1}, x_{2}, p_{c}=\frac{\hat{Q}}{z} ; \mu_{F}, \mu\right) D_{c \rightarrow \gamma^{*}(Q) X}\left(z, \mu_{F}^{2} ; Q^{2}\right) .
\end{aligned}
$$

In our modified factorization formalism, the LO contribution to the Drell-Yan cross section at large $Q_{T}$ is equal to the sum of the LO terms of the two perturbative expansions:

$$
\frac{d \sigma_{A B \rightarrow \gamma^{*}(Q) X}^{(L O)}}{d Q_{T}^{2} d y}=\frac{d \sigma_{A B \rightarrow \gamma^{*}(Q) X}^{(D i r-L O)}}{d Q_{T}^{2} d y}+\frac{d \sigma_{A B \rightarrow \gamma^{*}(Q) X}^{(F-L O)}}{d Q_{T}^{2} d y} .
$$

The LO direct and fragmentation contributions are found in Eq. (37) and Eq. (38), respectively.

At NLO, $\alpha_{e m} \alpha_{s}^{2}$, in the conventional fixed-order approach, the Drell-Yan cross section has the same form as that in Eq. (36) but with superscripts $(C-L O)$ replaced by $(C-N L O)$.

According to our modified factorization formalism, the NLO term should be the sum of the NLO terms in both the direct and fragmentation contributions in Eq. (35). The NLO direct contribution is 


$$
\begin{aligned}
\frac{d \sigma_{A B \rightarrow \gamma^{*}(Q) X}^{(D i r-N L)}}{d Q_{T}^{2} d y}= & \sum_{a, b} \int d x_{1} \phi_{a / A}\left(x_{1}, \mu\right) \int d x_{2} \phi_{b / B}\left(x_{2}, \mu\right) \\
\times & {\left[\frac{d \hat{\sigma}_{a b \rightarrow \gamma^{*}(Q) X}^{(C-N L O)}}{d Q_{T}^{2} d y}\left(x_{1}, x_{2}, Q, Q_{T}, y ; \mu\right)\right.} \\
& \left.-\sum_{c} \int \frac{d z}{z^{2}} \frac{d \hat{\sigma}_{a b \rightarrow c X}^{(F-L O)}}{d p_{c_{T}}^{2} d y}\left(x_{1}, x_{2}, p_{c}=\frac{\hat{Q}}{z} ; \mu_{F}, \mu\right) D_{c \rightarrow \gamma^{*}(Q) X}^{(0)}\left(z, \mu_{F}^{2} ; Q^{2}\right)\right] .
\end{aligned}
$$

The subtraction term is a consequence of the definition of the direct contribution in Eq. (34). The subtraction term is also necessary to remove the logarithmic terms from the conventional NLO expression in Eq. (40), to avoid double counting. These logarithmic contributions have been included in the LO fragmentation contribution to the Drell-Yan cross section in Eq. (39).

We would need NLO fragmentation contributions to complete the calculation of the NLO contribution,

$$
\begin{aligned}
\frac{d \sigma_{A B \rightarrow \gamma^{*}(Q) X}^{(F-N L O)}}{d Q_{T}^{2} d y}= & \sum_{a, b} \int d x_{1} \phi_{a / A}\left(x_{1}, \mu\right) \int d x_{2} \phi_{b / B}\left(x_{2}, \mu\right) \\
& \times \sum_{c} \int \frac{d z}{z^{2}} \frac{d \hat{\sigma}_{a b \rightarrow c X}^{(F-N L O)}}{d p_{c_{T}}^{2} d y}\left(x_{1}, x_{2}, p_{c}=\frac{\hat{Q}}{z} ; \mu_{F}, \mu\right) D_{c \rightarrow \gamma^{*}(Q) X}\left(z, \mu_{F}^{2} ; Q^{2}\right) .
\end{aligned}
$$

The virtual-photon fragmentation functions include the all orders resummation of the logarithmic terms, and they are the same as those for the LO contribution in Eq. (38). The $O\left(\alpha_{S}^{3}\right)$ partonic hard parts $d \hat{\sigma}_{a b \rightarrow c X}^{(F-N L O)} / d p_{c_{T}}^{2} d y$ are defined in Sec. III and will be presented elsewhere.

The NLO contribution to the Drell-Yan cross section at large transverse momentum is

$$
\frac{d \sigma_{A B \rightarrow \gamma^{*}(Q) X}^{(N L O)}}{d Q_{T}^{2} d y} \equiv \frac{d \sigma_{A B \rightarrow \gamma^{*}(Q) X}^{(D i r-N L O)}}{d Q_{T}^{2} d y}+\frac{d \sigma_{A B \rightarrow \gamma^{*}(Q) X}^{(F-N L O)}}{d Q_{T}^{2} d y}
$$

where the NLO direct and fragmentation contributions are found in Eqs. (40) and (41), respectively.

In our modified factorization formalism, the LO and NLO contributions are different from those in the conventional formalism because the virtual photon fragmentation functions include all orders of the large leading logarithmic contributions. The difference is better seen if we rewrite our LO and NLO results in the following form,

$$
\begin{aligned}
\frac{d \sigma_{A B \rightarrow \gamma^{*}(Q) X}}{d Q_{T}^{2} d y} \equiv & \frac{d \sigma_{A B \rightarrow \gamma^{*}(Q) X}^{(L O)}}{d Q_{T}^{2} d y}+\frac{d \sigma_{A B \rightarrow \gamma^{*}(Q) X}^{(N L O)}}{d Q_{T}^{2} d y} \\
= & \sum_{a, b} \int d x_{1} \phi_{a / A}\left(x_{1}, \mu\right) \int d x_{2} \phi_{b / B}\left(x_{2}, \mu\right) \\
& \times\left\{\frac{d \hat{\sigma}_{a b \rightarrow \gamma^{*}(Q) X}^{(C-L O)}}{d Q_{T}^{2} d y}\left(x_{1}, x_{2}, Q, Q_{T}, y ; \mu\right)+\frac{d \hat{\sigma}_{a b \rightarrow \gamma^{*}(Q) X}^{(C-N L)}}{d Q_{T}^{2} d y}\left(x_{1}, x_{2}, Q, Q_{T}, y ; \mu\right)\right\}
\end{aligned}
$$




$$
\begin{aligned}
& +\sum_{a, b} \int d x_{1} \phi_{a / A}\left(x_{1}, \mu\right) \int d x_{2} \phi_{b / B}\left(x_{2}, \mu\right) \\
& \times\left\{\sum_{c} \int \frac{d z}{z^{2}} \frac{d \hat{\sigma}_{a b \rightarrow c X}^{(F-L O)}}{d p_{c_{T}}^{2} d y}\left(x_{1}, x_{2}, p_{c}=\frac{\hat{Q}}{z} ; \mu_{F}, \mu\right)\right. \\
& \quad \times\left[D_{c \rightarrow \gamma^{*}(Q) X}\left(z, \mu_{F}^{2} ; Q^{2}\right)-D_{c \rightarrow \gamma^{*}(Q) X}^{(0)}\left(z, \mu_{F}^{2} ; Q^{2}\right)\right] \\
& \left.\quad+\sum_{c} \int \frac{d z}{z^{2}} \frac{d \hat{\sigma}_{a b \rightarrow c X}^{(F-N L O)}}{d p_{c_{T}}^{2} d y}\left(x_{1}, x_{2}, p_{c}=\frac{\hat{Q}}{z} ; \mu_{F}, \mu\right) D_{c \rightarrow \gamma^{*}(Q) X}\left(z, \mu_{F}^{2} ; Q^{2}\right)\right\} .
\end{aligned}
$$

On the right-hand-side of Eq. (44), the first term the prediction of the conventional fixedorder factorization formalism in Eq. (2); the second term is the difference between our modified factorization formula and the conventional fixed-order factorization formula up to NLO.

To conclude this section, we emphasize that our modified factorization formalism in Eq. (35) effectively reorganizes the single perturbative expansion of conventional QCD factorization, $d \hat{\sigma}_{a b \rightarrow \gamma^{*}(Q) X} / d Q_{T}^{2} d y$, in Eq. (2), into two perturbative expansions, $d \hat{\sigma}_{a b \rightarrow \gamma^{*}(Q) X}^{(D i r)} / d Q_{T}^{2} d y$ and $d \hat{\sigma}_{a b \rightarrow c X}^{(F)} / d p_{c_{T}}^{2} d y$, plus the perturbatively calculated evolution kernels. The main advantage of this reorganization is that the new perturbative expansions are evaluated at a single hard scale and are free of large logarithms. As shown in the next section, the ratios of the NLO over the LO contributions in the new perturbative expansions are smaller than the ratios evaluated in the conventional approach.

\section{NUMERICAL RESULTS AND PREDICTIONS}

In this section, we present numerical evaluations of the leading and next-to-leading order Drell-Yan cross section at large transverse momentum. We show the quantitative differences between the predictions of the modified factorization formula of Eq. (35) and the conventional factorization formula. We demonstrate the sensitivity of the cross section to the gluon distribution at low $Q^{2}$ and high $Q_{T}$.

We employ the CTEQ5M set of parton distributions [21]. We use a two-loop expression for the strong coupling strength $\alpha_{s}$, with the value of $\Lambda_{\mathrm{QCD}}$ specified by CTEQ5M, and a one loop expression for $\alpha_{e m}$ with $\alpha_{e m}\left(M_{Z}\right)=1 / 128$. We choose $Q^{2}$ as the renormalization scale for $\alpha_{e m}$. We equate the renormalization and factorization scales and set hard scale $\mu=\mu_{f}=\kappa \sqrt{Q_{T}^{2}+Q^{2}}$ with constant $\kappa=O(1)$.

The normal $\overline{M S}$ factorization scheme removes the ultraviolet $1 / \epsilon$ pole of the partonlevel fragmentation functions along with the corresponding splitting functions for massless partons, but the scheme does not guarantee that the fragmentation functions to a massive parton or to a photon with non-vanishing invariant mass [12] will be positive. As a result, the virtual photon fragmentation functions calculated in the $\overline{M S}$ scheme can be negative in the region of large $z$ [12,17]. As long as the cross section is positive, a negative fragmentation function is simply a particular separation of finite contributions between the coefficient function and the fragmentation function. It is not a problem in principle. Nevertheless, it is more appealing intuitively that the fragmentation functions be positive definite. Since the virtual photon fragmentation functions are purely perturbative, it is possible to preserve 
positivity of the fragmentation functions if we require that the mass threshold constraints be respected at every stage of the fragmentation (or bremsstrahlung radiation).

As shown in Ref. [12], the ultraviolet (UV) divergences of the virtual photon fragmentation functions come from two sources: (1) elementary divergent diagrams associated with the renormalization of the fields and coupling constants in QCD and QED, and (2) the loop momenta of the skeleton ladder diagrams. An invariant mass cutoff scheme is introduced in Ref. [12] to render the fragmentation functions positive definite. In this scheme, all UV divergences associated with the internal elementary divergent diagrams are removed in the same way as the QCD and QED Lagrangian are renormalized (say, in the $\overline{M S}$ scheme). The UV divergences connected with loop momenta of the skeleton ladder diagrams and wave function renormalization of the composite operators are removed by imposition of an invariant mass cut on the loop momenta. All running coupling constants in this scheme are renormalized in the same way as the renormalization of the Lagrangian. Use of the same invariant mass cutoff on the loop momenta of the skeleton ladder diagrams and the virtual diagrams due to the wave function renormalization of the composite operators ensures the infrared cancellation between the real and the virtual diagrams [16]. In this invariant mass cut-off scheme, a parton-to-virtual-photon fragmentation function $D_{c \rightarrow \gamma^{*}(Q) X}\left(z, \mu_{F}^{2} ; Q^{2}\right)$ can be viewed as an inclusive rate for "decay" of the parton of flavor $c$ and squared invariant mass $\mu_{F}^{2}$ into a virtual photon of squared invariant mass $Q^{2}$ and momentum fraction $z$ [12].

In our numerical calculations, we use the virtual photon fragmentation functions in the invariant mass cut-off scheme of Ref. 12]. The invariant mass for quark $c$ in Fig. 33 to decay into a virtual photon of invariant mass $Q$ and a massless quark is

$$
p_{c}^{2}=\frac{1}{z(1-z)} \vec{Q}_{T}^{2}+\frac{Q^{2}}{z} .
$$

The three-vector $\vec{Q}_{T}$ is perpendicular to the direction of the parent quark's momentum. The fragmentation scale is chosen to be the invariant mass of the fragmenting parton. For example, we choose $\mu_{F}^{2}=p_{c}^{2}$ at lowest order. Equation (45) shows that the mass threshold requires that the fragmentation scale be $\mu_{F}^{2} \geq Q^{2} / z$ [12,17]. If we keep the perturbative contributions to a high enough order, the cross section should not be sensitive to the choice of fragmentation scale. However, since the fragmentation scale in this new scheme is very different from the traditional scale in the $\overline{M S}$ scheme, we test two choices for the fragmentation scale,

$$
\begin{aligned}
& \mu_{F}=\kappa \sqrt{Q_{T}^{2}+Q^{2}}, \\
& \mu_{F}=\kappa \sqrt{\frac{Q_{T}^{2}+Q^{2}}{z}} .
\end{aligned}
$$

The first choice is the same as that for the renormalization and factorization scales.

The second choice in Eq. (47) is motivated by the fact that the squared invariant mass of the fragmenting parton is of the order $\left(Q_{T}^{2}+Q^{2}\right) / z$, obtained as follows. For the generic $2 \rightarrow 3$ diagram in Fig. 3, with the final parton that recoils against $p_{c}$ assumed massless, we compute

$$
\begin{aligned}
p_{c}^{2} & =2\left(p_{a}+p_{b}\right) \cdot p_{c}-\left(p_{a}+p_{b}\right)^{2} \\
& \approx-\frac{t+u}{z} .
\end{aligned}
$$


To derive the second line, we use the approximations $Q^{2} \ll Q_{T}^{2}$ and $s<|t+u| / z$. If we let $\mu_{F}^{2} \sim p_{c}^{2}$, Eq. (48) leads to the fragmentation logarithm,

$$
\ln \left(\frac{\mu_{F}^{2}}{Q^{2} / z}\right) \approx \ln \left(\frac{-(t+u) / z}{Q^{2} / z}\right)=\ln \left(\frac{-(t+u)}{Q^{2}}\right),
$$

consistent with the logarithm in Eq. (7). Since $|t+u| \sim O\left(Q_{T}^{2}+Q^{2}\right)$, we find $\mu_{F}^{2} \sim$ $\left(Q_{T}^{2}+Q^{2}\right) / z$.

The perturbatively calculated partonic parts in conventional perturbation theory, $\hat{\sigma}_{a b \rightarrow \gamma^{*}(Q) X}^{(C-L O)}$ and $\hat{\sigma}_{a b \rightarrow \gamma^{*}(Q) X}^{(C-N L O)}$ in Eq. (44), are available and calculated in the $\overline{\mathrm{MS}}$ scheme [2,7]. The fragmentation contribution in Eq. (38) depends on the leading order short-distance partonic parts, $\hat{\sigma}_{a b \rightarrow c X}^{(F-L O)}$, and on the virtual photon fragmentation functions. The partonic parts are

$$
\frac{\hat{\sigma}_{a b \rightarrow c X}^{(F-L O)}}{d p_{c_{T}}^{2} d y}=\frac{\pi}{2 x_{1} x_{2} S}\left|\frac{1}{g^{2}} \bar{M}_{a b \rightarrow c X}\right|^{2}\left(8 \pi^{2}\right) \delta\left(\left(x_{1} P_{A}+x_{2} P_{B}-p_{c}\right)^{2}\right)\left(\frac{\alpha_{s}(\mu)}{2 \pi}\right)^{2} .
$$

The functions $\bar{M}_{a b \rightarrow c X}$ are the lowest order matrix elements for partons $a$ and $b$ to produce a parton of flavor $c$, averaged over the colors and spins of the partons in the initial state. They are available in Ref. [13].

To compare with data, we introduce the invariant differential cross section

$$
\begin{aligned}
E \frac{d^{3} \sigma_{A B \rightarrow \ell^{+} \ell^{-} X}}{d^{3} Q} & =\frac{1}{\pi} \int d Q^{2}\left[\frac{d \sigma_{A B \rightarrow \ell^{+} \ell^{-}(Q) X}}{d Q^{2} d Q_{T}^{2} d y}\right] \\
& =\frac{\alpha_{e m}}{3 \pi^{2}} \int \frac{d Q^{2}}{Q^{2}}\left[\frac{d \sigma_{A B \rightarrow \gamma^{*}(Q) X}}{d Q_{T}^{2} d y}\right]
\end{aligned}
$$

The integration in $d Q^{2}$ is over a bin centered on the invariant mass $Q$. The differential cross sections $d \sigma_{A B \rightarrow \ell^{+} \ell^{-} X} / d Q^{2} d Q_{T}^{2} d y$ and $d \sigma_{A B \rightarrow \gamma^{*} X} / d Q_{T}^{2} d y$ are given in Eq. (1) and Eq. (43), respectively. If the bin size $\Delta Q$ is much smaller than $Q$, the cross section can be approximated as

$$
E \frac{d^{3} \sigma_{A B \rightarrow \ell^{+} \ell^{-} X}}{d^{3} Q} \approx \frac{2 \alpha_{e m}}{3 \pi^{2}} \frac{\Delta Q}{Q}\left[\frac{d \sigma_{A B \rightarrow \gamma^{*}(Q) X}}{d Q_{T}^{2} d y}\right]
$$

The differential cross section through next-to-leading order for production of a virtual photon, $d \sigma_{A B \rightarrow \gamma^{*}(Q) X} / d Q_{T}^{2} d y$, is given in Eq. (43).

Since we do not present the NLO partonic hard parts for the fragmentation contributions in this paper, we use Eq. (43) without the $d \hat{\sigma}_{a b \rightarrow c X}^{(F-N L O)} / d p_{c_{T}}^{2} d y$ term for the numerical calculations of the Drell-Yan cross sections.

In Fig. 7, 8, and 9, we plot the differential cross section $E d^{3} \sigma / d^{3} Q$ as a function of $Q_{T}$ for $p \bar{p} \rightarrow \ell^{+} \ell^{-}+X$ at the Tevatron collider energy $\sqrt{S}=2.0 \mathrm{TeV}$, for $p p \rightarrow \ell^{+} \ell^{-}+X$ at the LHC energy $\sqrt{S}=14 \mathrm{TeV}$, and for $p p \rightarrow \ell^{+} \ell^{-}+X$ at the RHIC proton-proton energy $\sqrt{S}=500 \mathrm{GeV}$. In all three figures, the rapidity $y=0$. The renormalization, factorization, and fragmentation scales are set equal, with constant $\kappa=1$. The solid, dashed, dotted, and dot-dashed lines represent the total, the LO direct, the NLO direct, and the resummed fragmentation contributions, respectively. We show results for virtual photon invariant mass 
$2 \leq Q \leq 3 \mathrm{GeV}$ and $4 \leq Q \leq 5 \mathrm{GeV}$. (Note: the solid curve represents the sum of the dashed, dotted, and dot-dashed lines.) To enhance the cross sections, the smaller value of the invariant mass $Q$ is favored as long as the lepton pairs can be identified experimentally.

At the integrated luminosities of run I of the Fermilab collider, prompt real photons have been observed with values of transverse momentum extending to $100 \mathrm{GeV}$ and beyond [22]. Scaling to the massive lepton pair case, we judge that it should be possible to examine cross sections in the same data sample out to $Q_{T}$ of $30 \mathrm{GeV}$ or more for virtual photon invariant mass $2 \leq Q \leq 3 \mathrm{GeV}$. Values of $Q_{T}$ to $50 \mathrm{GeV}$ or so may be reached with $2 \mathrm{fb}^{-1}$ at run II.

To demonstrate the renormalization, factorization and fragmentation scale-dependence of the cross sections, we introduce the ratio

$$
R_{\mu} \equiv E \frac{d \sigma_{A B \rightarrow \ell^{+} \ell^{-} X}}{d^{3} Q}(\kappa) / E \frac{d \sigma_{A B \rightarrow \ell^{+} \ell^{-} X}}{d^{3} Q}(\kappa=1) .
$$

The denominator in Eq. (53) is obtained with all three scales equal: $\mu=\mu_{f}=\mu_{F}=$ $\sqrt{Q_{T}^{2}+Q^{2}}$. In Fig. 10, we plot $R_{\mu}$ as a function of $Q_{T}$ at the Tevatron energy and $y=0$, for $2 \mathrm{GeV}$ and $5 \mathrm{GeV}$, with different scale choices. We first fix all three scales to be the same: $\mu=\mu_{f}=\mu_{F}=\kappa \sqrt{Q_{T}^{2}+Q^{2}}$, with $\kappa=2$ (solid) and $\kappa=1 / 2$ (dashed). The dotted lines correspond to the choice $\mu=\mu_{f}=\sqrt{Q_{T}^{2}+Q^{2}}$, and $\mu_{F}=\sqrt{\left(Q_{T}^{2}+Q^{2}\right) / z}$, as defined in Eq. (47). As shown in Fig. 10, the scale dependence is not great. It yields an uncertainty of about $15 \%$ for all reasonable values of $Q_{T}$ at the Tevatron energy. The effect of the different choice for the fragmentation scale in Eq. (47) is also small. The same features are preserved at the other collider energies.

To show the quantitative difference between the conventional fixed-order perturbative calculations and the calculations with all-orders resummation of the large logarithmic terms, we introduce the ratios

$$
R^{(L O)} \equiv\left[E \frac{d \sigma_{A B \rightarrow \ell^{+} \ell^{-} X}^{(D i r-L O)}}{d^{3} Q}+E \frac{d \sigma_{A B \rightarrow \ell^{+} \ell^{-} X}^{(F-L O)}}{d^{3} Q}\right] /\left[E \frac{d \sigma_{A B \rightarrow \ell^{+} \ell^{-} X}^{(C-L O)}}{d^{3} Q}\right]
$$

for the LO contributions, and

$R \equiv\left[E \frac{d \sigma_{A B \rightarrow \ell^{+} \ell^{-} X}^{(D i r-L O)}}{d^{3} Q}+E \frac{d \sigma_{A B \rightarrow \ell^{+} \ell^{-} X}^{(D i r-N O)}}{d^{3} Q}+E \frac{d \sigma_{A B \rightarrow \ell^{+} \ell^{-} X}^{(F-L O)}}{d^{3} Q}\right] /\left[E \frac{d \sigma_{A B \rightarrow \ell^{+} \ell^{-} X}^{(C-L O)}}{d^{3} Q}+E \frac{d \sigma_{A B \rightarrow \ell^{+} \ell^{-} X}^{(C-N L)}}{d^{3} Q}\right]$.

The ratio $R^{(L O)}$ is the ratio of the $\mathrm{LO}$ contributions in the two different factorization formalisms. Since the LO direct contribution is the same as the LO term in the conventional calculation, any deviation of $R^{(L O)}$ from unity measures the size of the logarithmic contributions. In Fig. 11, we plot the ratio $R^{(L O)}$ as a function of $Q_{T}$ at different energies for $2 \leq Q \leq 3 \mathrm{GeV}$ and $4 \leq Q \leq 5 \mathrm{GeV}$. The solid, dashed, and dotted lines represent the Tevatron, LHC, RHIC energies. Owing to the threshold behavior of the fragmentation function, the fragmentation contribution vanishes for $Q_{T} \sim Q$, and $R^{(L O)} \rightarrow 1$. The logarithmic contributions are very important at the LHC energy and less important at RHIC energies. The reason for this energy dependence is the phase space penalty associated with the large 
invariant mass of the virtual photon. The large logarithm in the fragmentation function is proportional to $\ln \left(z \mu_{F}^{2} / Q^{2}\right)$ for each power of $\alpha_{s}$. Since the strong coupling strength $\alpha_{s}(\mu)$ is proportional to $1 / \ln \left(\mu^{2} / \Lambda_{\mathrm{QCD}}^{2}\right)$, the product

$$
\alpha_{s}(\mu) \ln \left(z \mu_{F}^{2} / Q^{2}\right) \propto \frac{\ln \left(z \mu_{F}^{2} / Q^{2}\right)}{\ln \left(\mu^{2} / \Lambda_{\mathrm{QCD}}^{2}\right)}
$$

becomes of order unity only when $\mu_{F} \sim \mu \gg Q^{2}$. Otherwise, the combination in Eq. (56) is not large because of the factors $z$ and $Q^{2}$ from the mass threshold.

The ratio $R$ in Eq. (55) measures the difference between the conventional calculation up to NLO accuracy and our resummed calculation, obtained from our modified factorization formalism without the NLO fragmentation contributions. Without the NLO fragmentation contributions $E d \sigma^{(F-N L O)} / d^{3} Q$ in the numerator, the ratio $R$ does not represent the entire ratio of the NLO contributions in the two different factorization approaches. Nevertheless, this ratio does indicate some of the effects of QCD resummation.

The difference between the numerator and the denominator of the ratio $R$ in Eq. (55) is determined entirely by the second term in Eq. (44) without the NLO fragmentation contributions,

$$
\begin{aligned}
& \sum_{a, b, c} \int d x_{1} \phi_{a / A}\left(x_{1}, \mu\right) \int d x_{2} \phi_{b / B}\left(x_{2}, \mu\right) \int \frac{d z}{z^{2}} \frac{d \hat{\sigma}_{a b \rightarrow c X}^{(F-L O)}}{d p_{c_{T}}^{2} d y}\left(x_{1}, x_{2}, p_{c}=\frac{\hat{Q}}{z} ; \mu_{F}, \mu\right) \\
& \quad \times\left[D_{c \rightarrow \gamma^{*}(Q) X}\left(z, \mu_{F}^{2} ; Q^{2}\right)-D_{c \rightarrow \gamma^{*}(Q) X}^{(0)}\left(z, \mu_{F}^{2} ; Q^{2}\right)\right] .
\end{aligned}
$$

This difference is proportional to the difference between the LO QED parton-to-virtualphoton fragmentation functions $D_{c \rightarrow \gamma^{*}(Q) X}^{(0)}\left(z, \mu_{F}^{2} ; Q^{2}\right)$ and the corresponding QCD evolved parton-to-virtual-photon fragmentation functions $D_{c \rightarrow \gamma^{*}(Q) X}\left(z, \mu_{F}^{2} ; Q^{2}\right)$. As shown in Ref. [12], one of the major differences between these fragmentation functions is the behavior at large $z$ :

$$
\begin{aligned}
& D_{c \rightarrow \gamma^{*}(Q) X}^{(0)}\left(z \rightarrow 1, \mu_{F}^{2} ; Q^{2}\right) \neq 0 \\
& D_{c \rightarrow \gamma^{*}(Q) X}\left(z \rightarrow 1, \mu_{F}^{2} ; Q^{2}\right)=0 .
\end{aligned}
$$

QCD evolution reduces the fragmentation function at large $z$ while it increases the fragmentation function at small $z$. If the cross section is dominated by the small (large) $z$ region, the numerator of the ratio $R$ in Eq. (55) is larger (smaller) than the denominator calculated in fixed-order perturbation theory.

The $z$-integration of the Drell-Yan cross section runs from $z_{\min }$ to 1 . We introduce a cutoff $z_{c}$ to limit the integration to the range $z_{\text {min }}$ to $z_{c}$, and we define the ratio

$$
R_{z} \equiv E \frac{d \sigma_{A B \rightarrow \ell^{+} \ell^{-} X}^{(F-L O)}}{d^{3} Q}\left(z_{c}\right) / E \frac{d \sigma_{A B \rightarrow \ell^{+} \ell^{-} X}^{(F-L O)}}{d^{3} Q}\left(z_{c}=1\right)
$$

The cutoff $z_{c}$ can be between $z_{\min }$ and $1 ; R_{z}\left(z_{c}=z_{\min }\right)=0$, and $R_{z}\left(z_{c}=1\right)=1$. The shape of the ratio $R_{z}$ establishes which region of $z$ dominates the $z$-integration.

In Fig. 12, we plot $R_{z}$ as a function of $z_{c}$ at the Tevatron energy with $Q=2 \mathrm{GeV}$. The solid and dashed lines correspond to transverse momenta $Q_{T}=5$ and $50 \mathrm{GeV}$. If the 
integrand for the $z$-integration were independent of $z$, the ratio $R_{z}$ would be proportional to $\left(z_{c}-z_{\min }\right) /\left(1-z_{\min }\right)$, which corresponds to a straight line for $R_{z}$. The shape of $R_{z}$ in Fig. 12 shows that the $z$-integration is dominated by the large $z$ region.

Since the $z$-dependence of the partonic cross section in Eq. (38) tends to cancel the $1 / z^{2}$ factor in the $z$-integration, the $z$-integration for the Drell-Yan cross section is determined mainly by the convolution of parton distributions and fragmentation functions and by the shapes of these functions.

For a given collision energy $\sqrt{S}$, the transverse momentum $Q_{T}=z p_{c_{T}} \propto z x \sqrt{S}$ with $x \sim x_{1} \sim x_{2}$ for $y \simeq 0$. For a fixed value of $Q_{T}$, a large (small) $z$ corresponds to the small (large) $x$ region. Because the parton distributions and fragmentation functions are both steeply falling functions of the momentum fractions ( $x$ or $z$ ), and the Drell-Yan cross section is proportional to two powers of parton distributions and one power of fragmentation function, the convolution of parton distributions and fragmentation functions favors the combination of a small $x$ and large $z$. The fragmentation contributions are dominated by the small $x$ and large $z$ region. The $z$-integration over the difference between the QCD evolved fragmentation function and the leading order QED fragmentation function in Eq. (57) is dominated by the large $z$ region. The net contribution from this term is expected to be negative, and the ratio $R$ in Eq. (55) should be less than one for $Q_{T} \gg Q$.

We plot the ratio $R$ in Fig. 13 as a function of $Q_{T}$ at $y=0$ for $2 \leq Q \leq 3 \mathrm{GeV}$ and $4 \leq Q \leq 5 \mathrm{GeV}$ at different collision energies. The solid, dashed, and dotted lines in Fig. 13 correspond to the Tevatron energy $\sqrt{S}=2.0 \mathrm{TeV}$, the LHC energy $\sqrt{S}=14 \mathrm{TeV}$, and the RHIC proton-proton energy $\sqrt{S}=500 \mathrm{GeV}$, respectively. The ratio $R$ is less than one when $Q_{T}>Q$, as expected. As $Q_{T}$ grows, $z_{\min }$ becomes larger, and the fragmentation contributions are dominated by even larger values of $z$. Consequently, $R$ is a decreasing function of the transverse momentum $Q_{T}$. The predicted distribution in $Q_{T}$ is steepened relative to the conventional expectation, with the steepening being greater for smaller $\sqrt{S}$. The shape of the ratio $R$ represents the effect of QCD evolution on the virtual photon fragmentation functions. As $Q_{T} \rightarrow Q, R \rightarrow 1$, in keeping with the expectation that our modified factorization formula should yield the same cross section as conventional fixed-order perturbation theory when $Q_{T} \sim Q$. The apparent slight difference of $R$ from 1 near $Q_{T}=Q$ may be attributed to the choice of fragmentation scale $\mu_{F}$. If we were to set $\mu_{F}^{2}=Q_{T}^{2} / z$, our result would be the same as the conventional one when $Q_{T}=Q$.

The main advantages of our modified factorization formula in Eq. (35) are that the large logarithms are removed from the coefficient functions of conventional factorization and all perturbatively calculated coefficient functions are evaluated at a single hard scale. The reliability of the perturbative calculations is enhanced. In order to demonstrate this benefit, we introduce the ratios

$$
\begin{aligned}
R_{c} & \equiv E \frac{d \sigma_{A B \rightarrow \ell^{+} \ell^{-} X}^{(C-N L O)}}{d^{3} Q} / E \frac{d \sigma_{A B \rightarrow \ell^{+} \ell^{-} X}^{(C-L O)}}{d^{3} Q}, \\
R_{D i r} & \equiv E \frac{d \sigma_{A B \rightarrow \ell^{+} \ell^{-X}}^{(D i r-N L)}}{d^{3} Q} / E \frac{d \sigma_{A B \rightarrow \ell^{+} \ell^{-} X}^{(D i r-L O)}}{d^{3} Q}, \\
R_{F} & \equiv E \frac{d \sigma_{A B \rightarrow \ell^{+} \ell^{-} X}^{(F-N L O)}}{d^{3} Q} / E \frac{d \sigma_{A B \rightarrow \ell^{+} \ell^{-} X}^{(F-L O)}}{d^{3} Q} .
\end{aligned}
$$

The subscripts $c, D i r$, and $F$ represent the conventional perturbative calculation, our direct 
contribution, and our fragmentation contribution, respectively. Because the large logarithms are removed in the perturbatively calculated coefficient functions in the modified factorization formula, we expect the ratios $R_{D i r}$ and $R_{F}$ to be smaller than the ratio $R_{c}$.

We compare $R_{D i r}$ and $R_{c}$ in Figs. 14 and 15 at the Tevatron and LHC energies. As expected, $R_{D i r}$ is smaller than $R_{c}$. We remind the reader that in our notation, NLO corresponds to the pure $O\left(\alpha_{s}^{2}\right)$ contribution and LO corresponds to pure $O\left(\alpha_{s}\right)$. Thus, the commonly defined $K$-factor is obtained by adding 1 to the ratios Figs. 14 and 15. The factor $K$ would be near 2.3 for $Q_{T} \sim 10 \mathrm{GeV}$ (near 1.9 for $Q_{T} \sim 50 \mathrm{GeV}$ ) at $\sqrt{S}=2 \mathrm{TeV}$ and $2 \leq Q \leq 3 \mathrm{GeV}$ in the conventional approach, but somewhat smaller, $\sim 2$ and $\sim 1.6$, for our direct contribution. The relatively smaller role of the NLO term lends greater confidence in the reliability of the calculated Drell-Yan cross sections in our modified formalism.

The denominators are identical in the definitions of the ratios $R_{c}$ and $R_{D i r}$ in Eqs. (60) and (61). The difference between the solid and dashed curves in Figs. 14 and 15 is therefore the ratio of the logarithmic piece in NLO to the leading-order direct contribution. The figures show that a considerable fraction of the NLO correction in conventional perturbative calculations comes from logarithmic contributions associated with bremsstrahlung radiation of the virtual photon. As noted above, the NLO fragmentation contributions are not available yet, and, therefore, we do not show results here for $R_{F}$.

Taken at face value, the ratio $R$ in Fig. 13 suggests that the cross section predicted in our modified formalism is modestly less than that in the conventional approach for $Q_{T}>Q$ and that the shape of the predicted $Q_{T}$ distribution is steeper. However, we remind the reader that we have not included the NLO fragmentation contributions. When these are added in subsequent work, the predicted Drell-Yan cross sections from our modified factorization formula should be larger than those from the conventional NLO perturbative approach. Estimating the ratio of the fragmentation contributions by the ratio of the direct contributions, $R_{F} \sim R_{D i r}$, we expect the Drell-Yan cross sections, with the NLO fragmentation contributions included, will be greater by 10 to $30 \%$ than the predicted totals in Figs. 0 - 9. For example, at $\sqrt{S}=2 \mathrm{TeV}$ and $2 \leq Q \leq 3 \mathrm{GeV}$, we estimate increases of $15 \%$ and $10 \%$ at $Q_{T}=9$ and $39 \mathrm{GeV}$.

To demonstrate the relative size of gluon initiated contributions, we define the ratio

$$
R_{g}=\frac{d \sigma_{A B \rightarrow \gamma^{*}(Q) X} \text { (gluon-initiated) }}{d Q_{T}^{2} d y} / \frac{d \sigma_{A B \rightarrow \gamma^{*}(Q) X}}{d Q_{T}^{2} d y} .
$$

The numerator includes the contributions from all partonic subprocesses with at least one initial-state gluon, and the denominator includes all subprocesses. In Fig. 16, we show $R_{g}$ as a function of $Q_{T}$ at $y=0$ for the Tevatron energy (solid), the LHC energy (dashed), and the RHIC proton-proton energy (dotted) at $Q=2 \mathrm{GeV}$ and $Q=5 \mathrm{GeV}$. We confirm that gluon initiated subprocesses dominate the Drell-Yan cross section and that low mass Drell-Yan lepton-pair production at large transverse momentum is an excellent source of information on the gluon distribution [2]. The falloff of $R_{g}$ at very large $Q_{T}$ is related to the reduction of phase space and the fact that cross sections are evaluated at larger values of the partons' momentum fractions. In the Tevatron case, quark-antiquark subprocesses are expected to (re)assert their dominance at very large $Q_{T}$ because of the valence nature of the antiquark density in the incident antiproton. 


\section{CONCLUSIONS}

In this paper we introduce a new factorization formalism for the Drell-Yan cross section at large $Q_{T}$. It incorporates all-orders resummation of large logarithmic contributions into parton-to-virtual photon fragmentation functions. In addition to the logarithmic contributions, our expression for the cross section includes the usual non-logarithmic contributions, referred to often as direct contributions, in both leading $O\left(\alpha_{s}\right)$ and higher orders. This modified factorization formula agrees with the conventional fixed-order QCD expression when $Q_{T}^{2} \sim Q^{2}$. The difference between the modified factorization formula and the conventional formula is determined by QCD evolution of the virtual photon fragmentation functions. Our modification reorganizes the single perturbative expansion of conventional QCD factorization into two expansions plus the perturbatively calculated parton-to-virtual photon fragmentation functions. The new expansions are evaluated at a single hard scale and are free of large $\ln ^{m}\left(Q_{T}^{2} / Q^{2}\right)$ terms when $Q_{T}^{2} \gg Q^{2}$. The ratio of the next-to-leading $O\left(\alpha_{s}^{2}\right)$ to leading order $O\left(\alpha_{s}\right)$ contributions is smaller in the new expansion than in the conventional case. The reliability of the predictions is enhanced.

As shown in Fig. 11, the contributions from the logarithmic terms are important. They can be as large as $70 \%$ of the non-logarithmic contributions at the LHC energies. At RHIC energies, the logarithmic contributions are smaller because phase space at large transverse momentum is limited and the penalty associated with large invariant mass $Q$ is felt more strongly. In Fig. 7, 8, and 9, we present predictions through next-to-leading order at energies of interest for experiments at the Fermilab Tevatron, Brookhaven's RHIC, and the CERN Large Hadron Collider. Resummation somewhat steepens the shape of the predicted $Q_{T}$-spectrum relative to the conventional fixed-order approach. However, at the order in perturbation theory at which we work, it has only a modest effect on the normalization. We confirm that the Drell-Yan cross section at large $Q_{T}$ remains an excellent source of constraints on the gluon parton density.

\section{ACKNOWLEDGMENTS}

Work in the High Energy Physics Division at Argonne National Laboratory is supported by the U.S. Department of Energy, Division of High Energy Physics, Contract W-31-109ENG-38. The research of J-W Q and X-F Z at Iowa State University is supported in part by the U.S. Department of Energy under Grant No. DE-FG02-87ER40731. 


\section{REFERENCES}

[1] S. D. Drell and T. M. Yan, Phys. Rev. Lett. 25, 316 (1970); H. D. Politzer, Nucl. Phys. B129, 301 (1977).

[2] E. L. Berger, L. E. Gordon, and M. Klasen, Phys. Rev. D58, 074012 (1998).

[3] E. L. Berger and J.-W. Qiu, Phys. Lett. B248, 371 (1990); Phys. Rev. D44, 2002 (1991).

[4] E. L. Berger, X.-F. Guo, and J.-W. Qiu, Phys. Rev. Lett. 76, 2234 (1996); Phys. Rev. D54, 5470 (1996).

[5] J. C. Collins, D. E. Soper, and G. Sterman, Phys. Lett. 109B, 388 (1982); 126B, 275 (1983); 134B, 263 (1984); and in Perturbative Quantum Chromodynamics, edited by A. H. Mueller (World Scientific, Singapore, 1989).

[6] G. T. Bodwin, S. J. Brodsky, and G. P. Lepage, Phys. Rev. Lett. 47, 1799 (1981); Phys. Rev. D31, 2616 (1985); D34, 3932(E) (1986).

[7] R. K. Ellis, G. Martinelli, and R. Petronzio, Nucl. Phys. B211, 106 (1983); P. Arnold and M. H. Reno, ibid. B319, 37 (1989); B330, 284(E) (1990); P. Arnold, R. K. Ellis, and M. H. Reno, Phys. Rev. D40, 912 (1989); R. Gonsalves, J. Pawlowski, and C.-F. Wai, ibid. D40, 2245 (1989).

[8] C. Albajar et al., UA1 Collaboration, Phys. Lett. B209, 397 (1988).

[9] J. C. Collins, D. E. Soper, and G. Sterman, Nucl. Phys. B250, 199 (1985).

[10] R. K. Ellis and S. Veseli, Nucl. Phys. B511, 649 (1998).

[11] J.-W. Qiu and X.-F. Zhang, Phys. Rev. Lett. 86, 2724 (2001); Phys. Rev. D63, 114011 (2001); and references therein.

[12] J.-W. Qiu and X.-F. Zhang, Phys. Rev. D (in press) hep-ph/0101004.

[13] J. F. Owens, Rev. Mod. Phys. 59, 465 (1987); H. Baer, J. Ohnemus, and J. F. Owens, Phys. Rev. D42, 61 (1990).

[14] P. Aurenche, R. Baier, M. Fontannaz, Phys. Rev. D42, 1440 (1990); P. Aurenche et al., Nucl. Phys. B399, 34 (1993).

[15] R. K. Ellis, G. Martinelli, and R. Petronzio, Nucl. Phys. B211, 106 (1983).

[16] J. C. Collins and J.-W. Qiu, Phys. Rev. D39, 1398 (1989).

[17] E. Braaten and J. Lee, hep-ph/0102130.

[18] G. Curci, W. Furmanski, and R. Petronzio, Nucl. Phys. B175, 27 (1980).

[19] P. Aurenche, P. Chiappetta, M. Fontannaz, J. Ph. Guillet, and E. Pilon, Nucl. Phys. B399, 34 (1993), and references therein.

[20] G. Sterman et al., CTEQ Collaboration, Rev. Mod. Phys. 67, 157 (1995).

[21] H. L. Lai et al., Eur. Phys. J. C12, 375 (2000).

[22] CDF Collaboration, F. Abe et al, Phys. Rev. D48, 2998 (1993); Phys. Rev. Lett. 73, 2662 (1994); D0 Collaboration, S. Abachi et al, ibid. 77, 5011 (1996). 


\section{FIGURES}

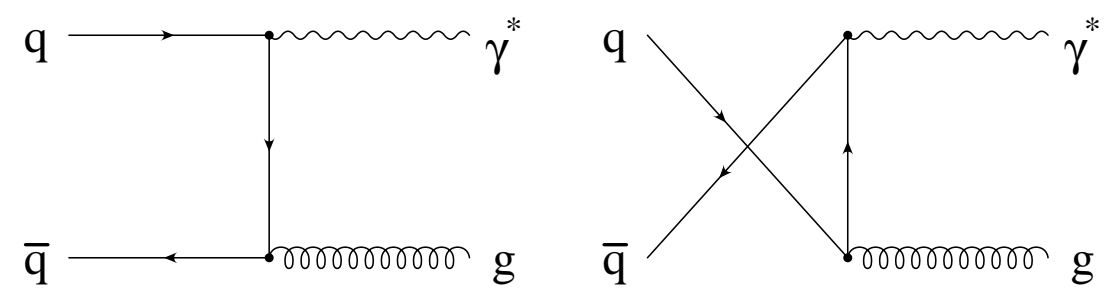

(a)
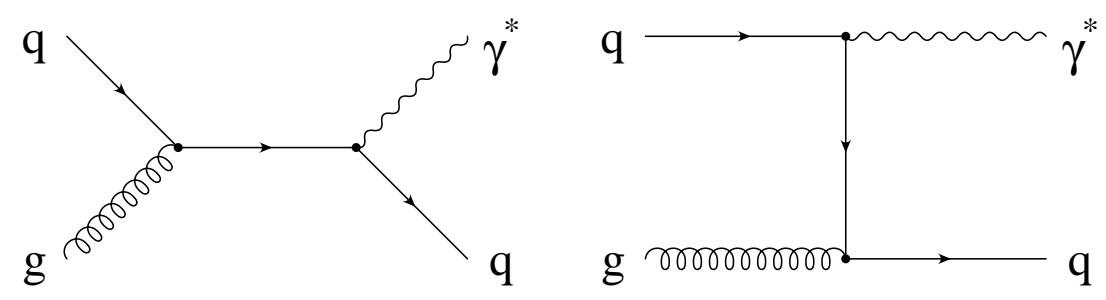

(b)

FIG. 1. Feynman diagrams for the LO contribution to the Drell-Yan cross section: (a) quark-antiquark annihilation $q+\bar{q} \rightarrow \gamma^{*}+g$, and (b) Compton $g+q \rightarrow \gamma^{*}+q$ subprocesses.
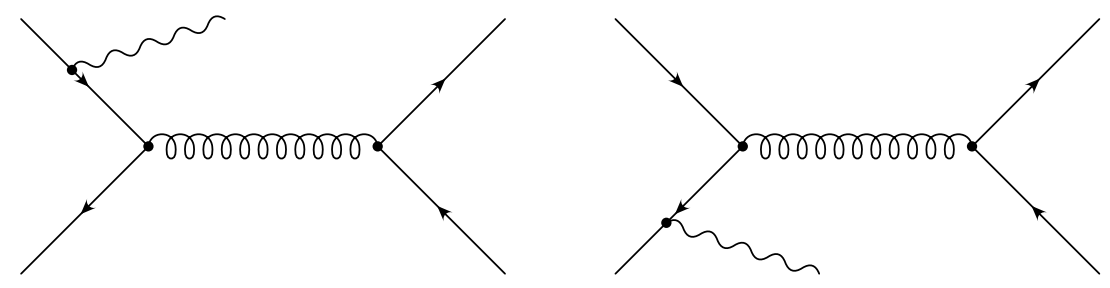

(a)
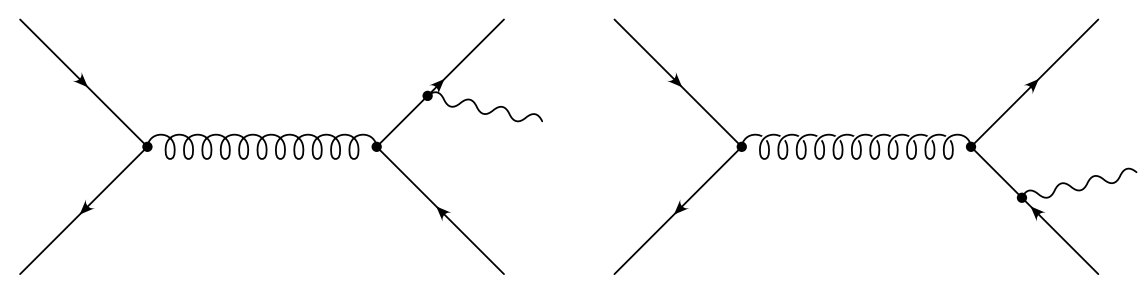

(b)

FIG. 2. Feynman diagrams that illustrate situations in which the photon can become collinear to a quark in (a) the initial-state and (b) the final-state. 


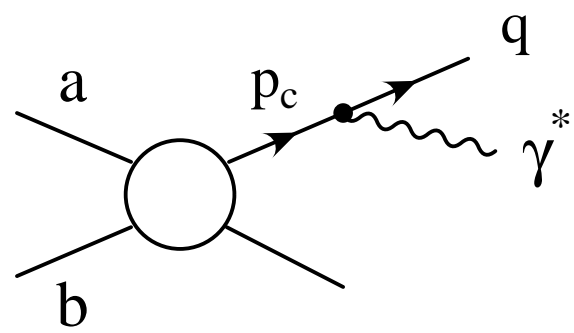

FIG. 3. A generic diagram of the lowest order $2 \rightarrow 3$ subprocess that contributes to the Drell-Yan cross section with large final-state logarithmic terms when $Q^{2} \ll Q_{T}^{2}$.

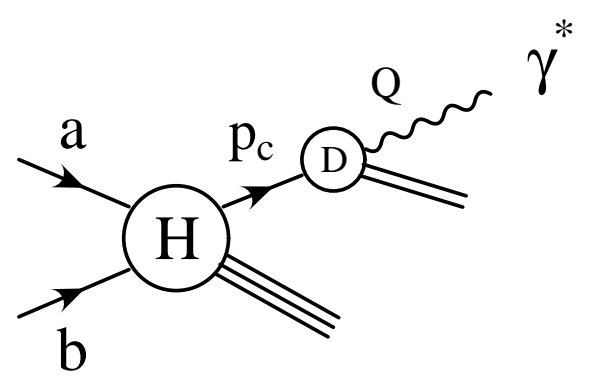

FIG. 4. Sketch of the fragmentation contribution to low mass Drell-Yan lepton-pair production at high $Q_{T}$.

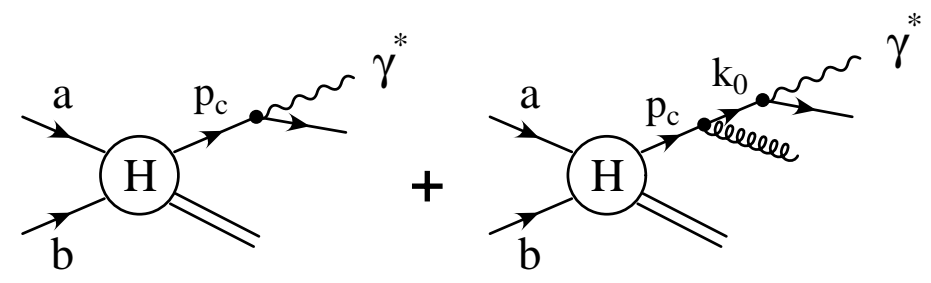

(a)

(b)

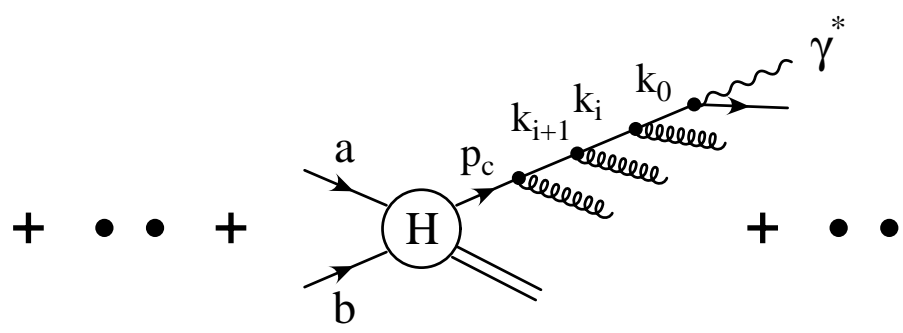

(c)

FIG. 5. Scattering amplitudes that provide large logarithmic contributions to the Drell-Yan cross section via quark fragmentation. 


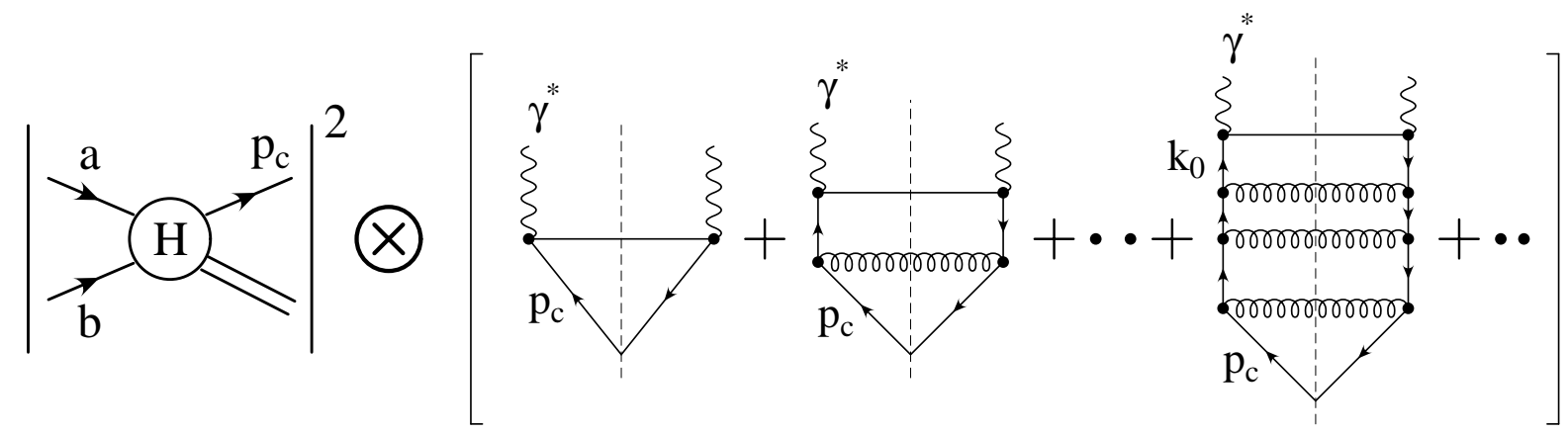

FIG. 6. Factored non-singlet quark-to-virtual-photon fragmentation contributions to the Drell-Yan cross section.
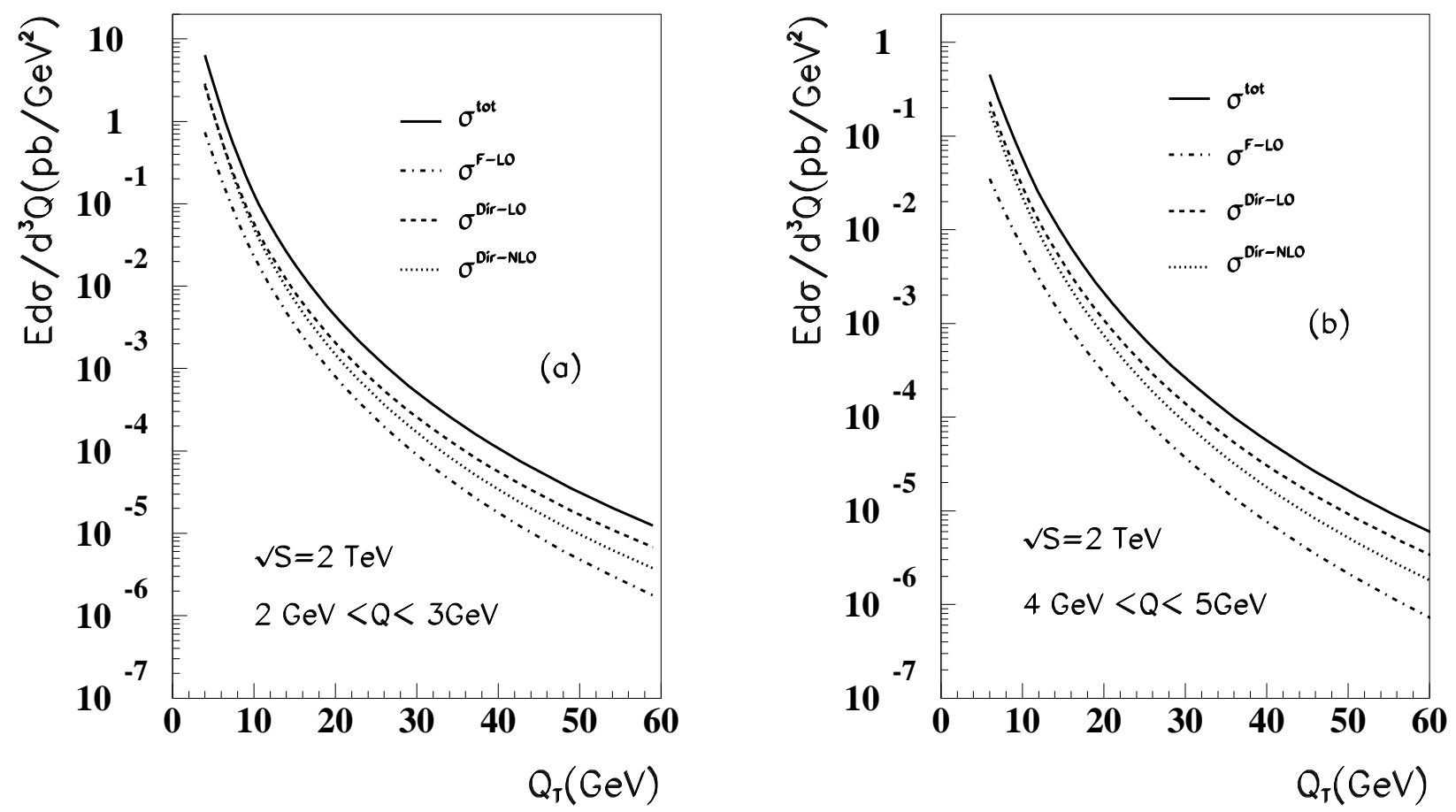

FIG. 7. Drell-Yan cross section as a function of $Q_{T}$ at the Tevatron energy $\sqrt{S}=2.0 \mathrm{TeV}$ and rapidity $y=0$ for the mass intervals (a) $2 \leq Q \leq 3 \mathrm{GeV}$ and (b) $4 \leq Q \leq 5 \mathrm{GeV}$. Solid, dashed, dotted, and dot-dashed lines stand for the total, LO direct, NLO direct, and resummed fragmentation contributions, respectively. 

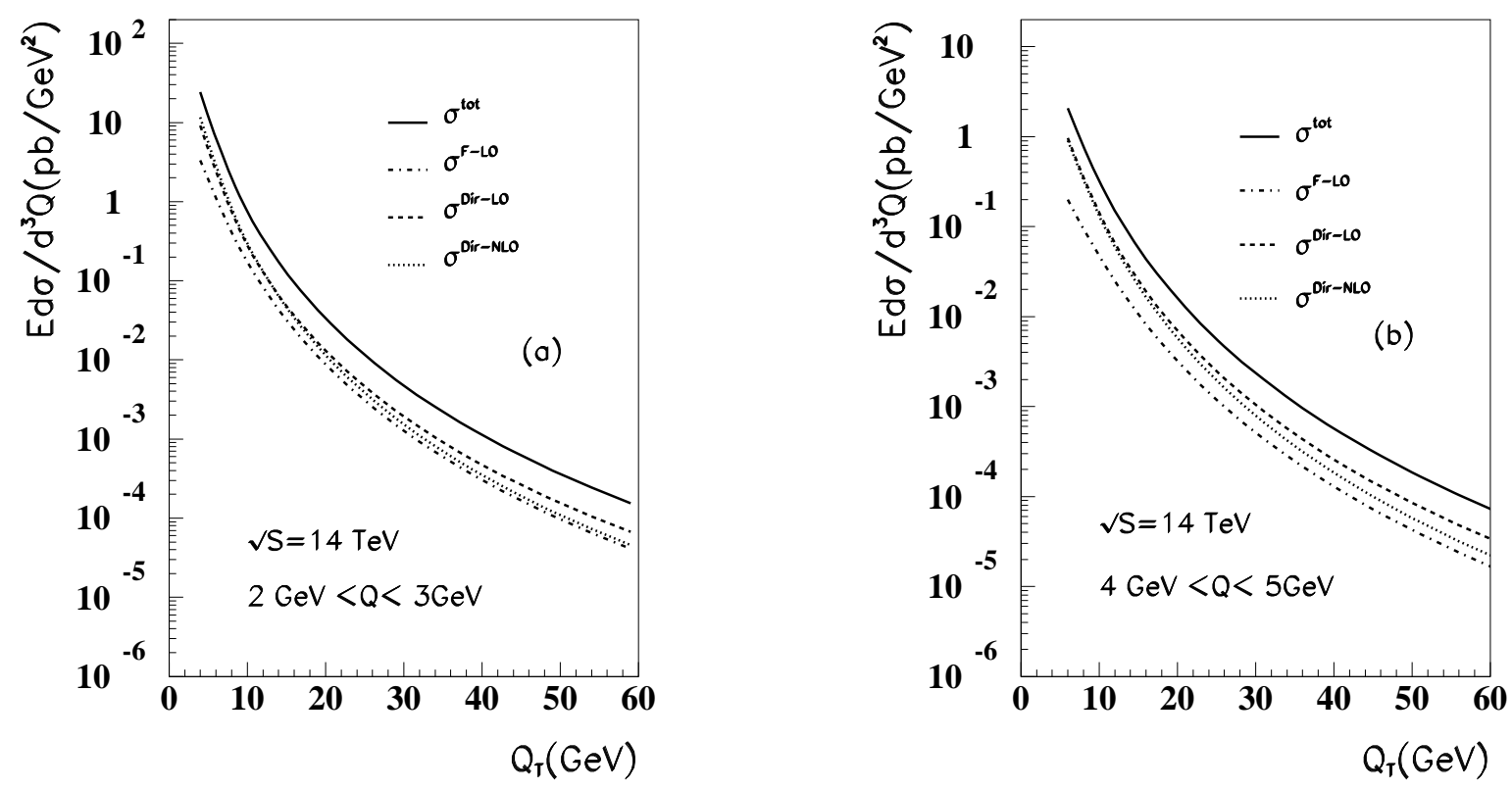

FIG. 8. Drell-Yan cross section as a function of $Q_{T}$ at the LHC energy $\sqrt{S}=14 \mathrm{TeV}$ and rapidity $y=0$ for the mass intervals (a) $2 \leq Q \leq 3 \mathrm{GeV}$ and (b) $4 \leq Q \leq 5 \mathrm{GeV}$. Solid, dashed, dotted, and dot-dashed lines stand for the total, LO direct, NLO direct, and resummed fragmentation contributions, respectively.
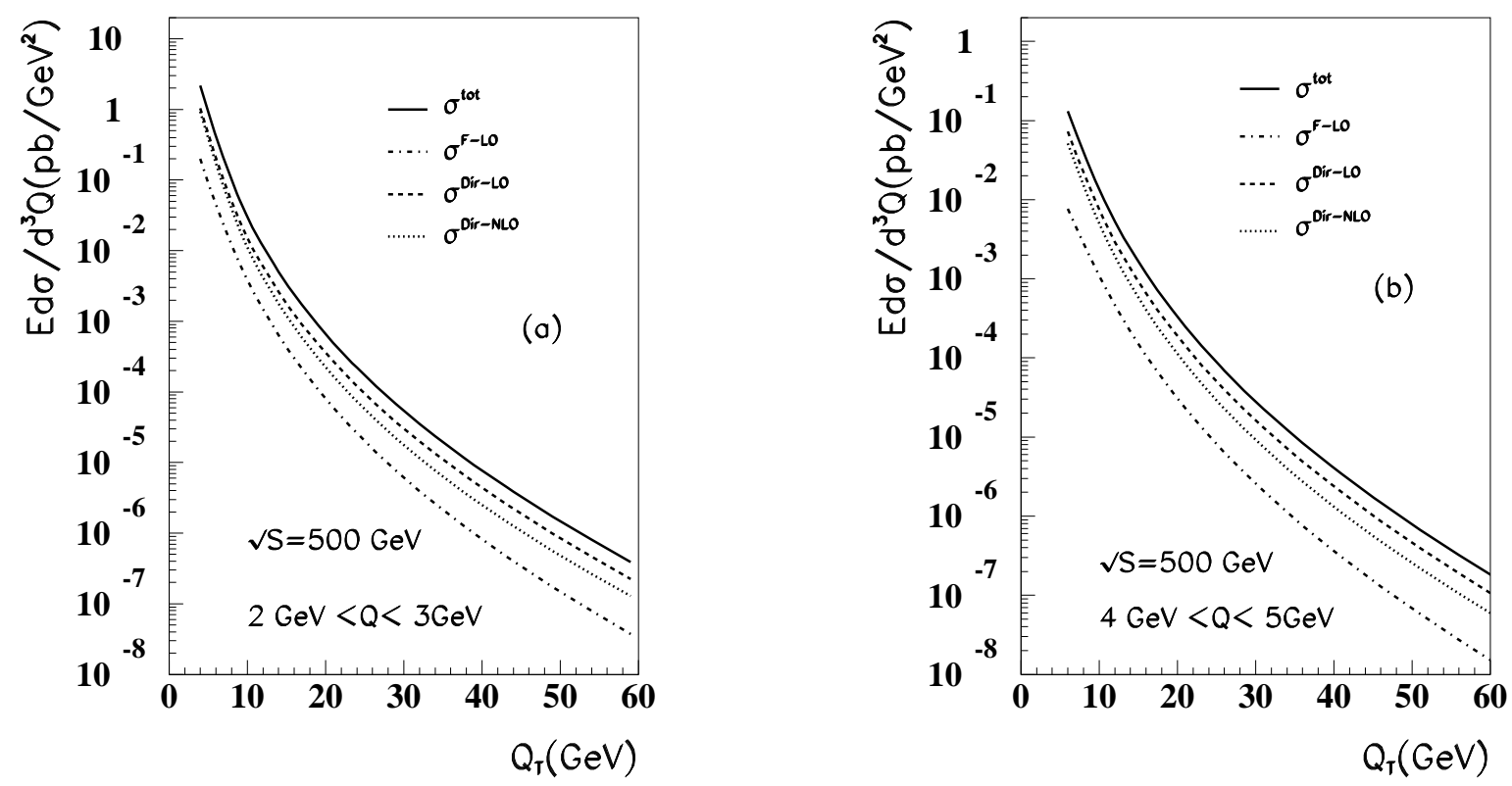

FIG. 9. Drell-Yan cross section as a function of $Q_{T}$ at the RHIC proton-proton energy $\sqrt{S}=500 \mathrm{GeV}$ and rapidity $y=0$ for the mass intervals (a) $2 \leq Q \leq 3 \mathrm{GeV}$ and (b) $4 \leq Q \leq 5 \mathrm{GeV}$. Solid, dashed, dotted, and dot-dashed lines stand for the total, LO direct, NLO direct, and resummed fragmentation contributions, respectively. 

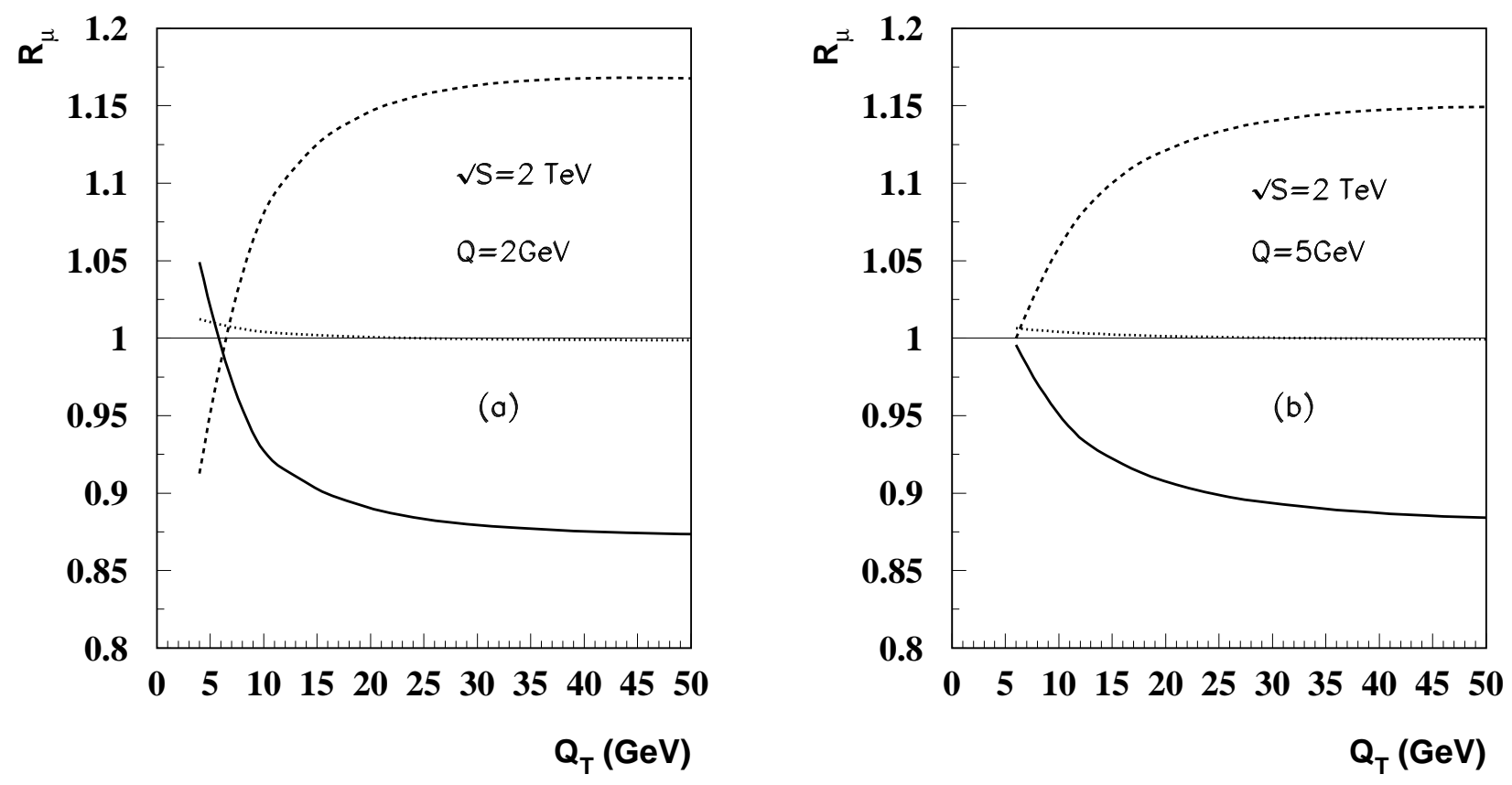

FIG. 10. Ratio $R_{\mu}$ in Eq. (53) as a function of $Q_{T}$ at $y=0$ and the Tevatron energy $\sqrt{S}=2 \mathrm{TeV}$ for virtual photon invariant mass (a) $2 \mathrm{GeV}$ and (b) $5 \mathrm{GeV}$. The curves are explained in the text.
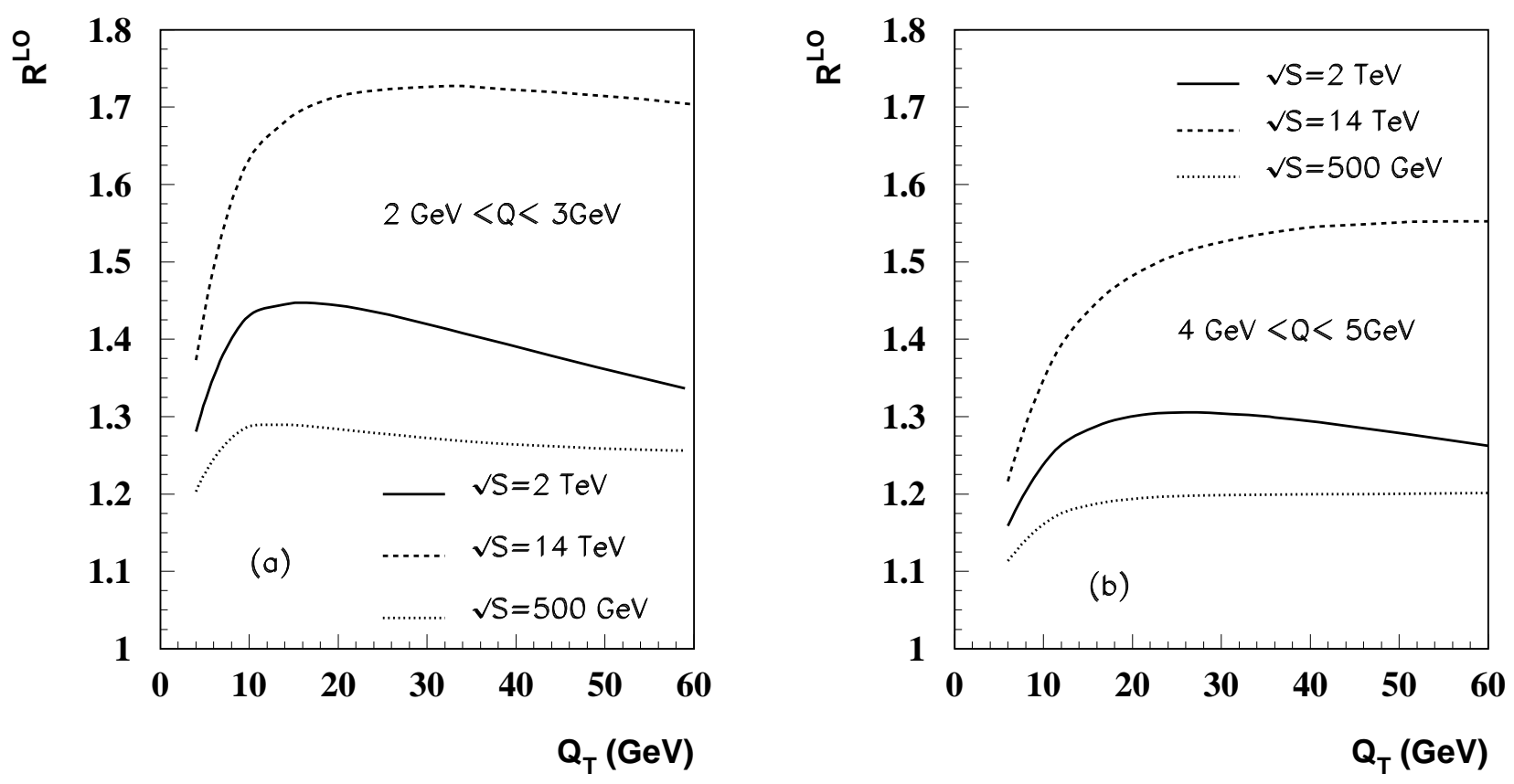

FIG. 11. Ratio $R^{(L O)}$ in Eq. (54) as a function of $Q_{T}$ at $y=0$ and three different collision energies for the mass intervals (a) $2 \leq Q \leq 3 \mathrm{GeV}$ and (b) $4 \leq Q \leq 5 \mathrm{GeV}$. The curves are explained in the text. 


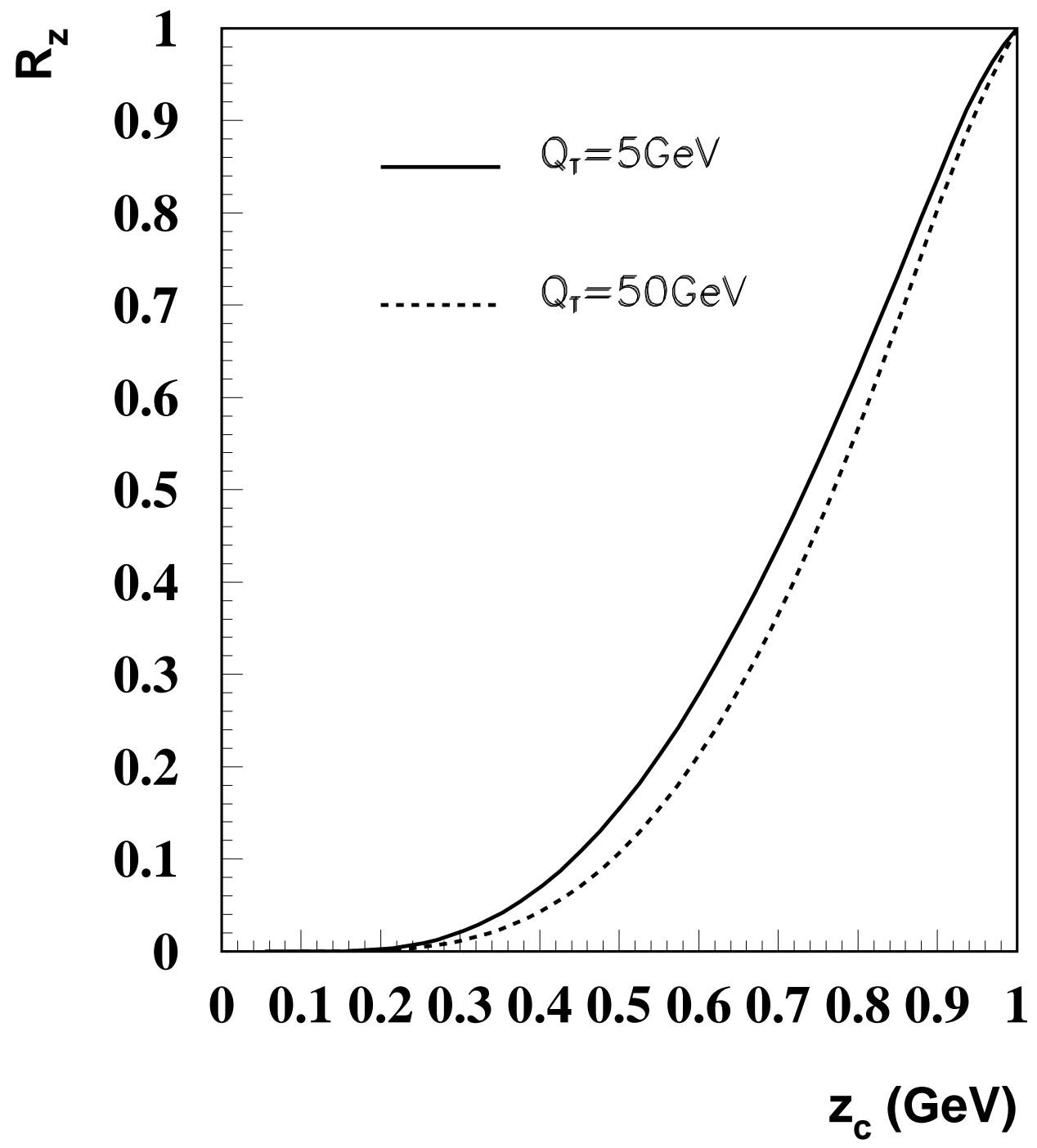

FIG. 12. Ratio $R_{z}$ in Eq. (59) as a function of $z_{c}$ at the Tevatron energy $\sqrt{S}=2 \mathrm{TeV}, y=0$, and $Q=2 \mathrm{GeV}$. The solid and dashed lines correspond to transverse momenta $Q_{T}=5$ and $50 \mathrm{GeV}$. 

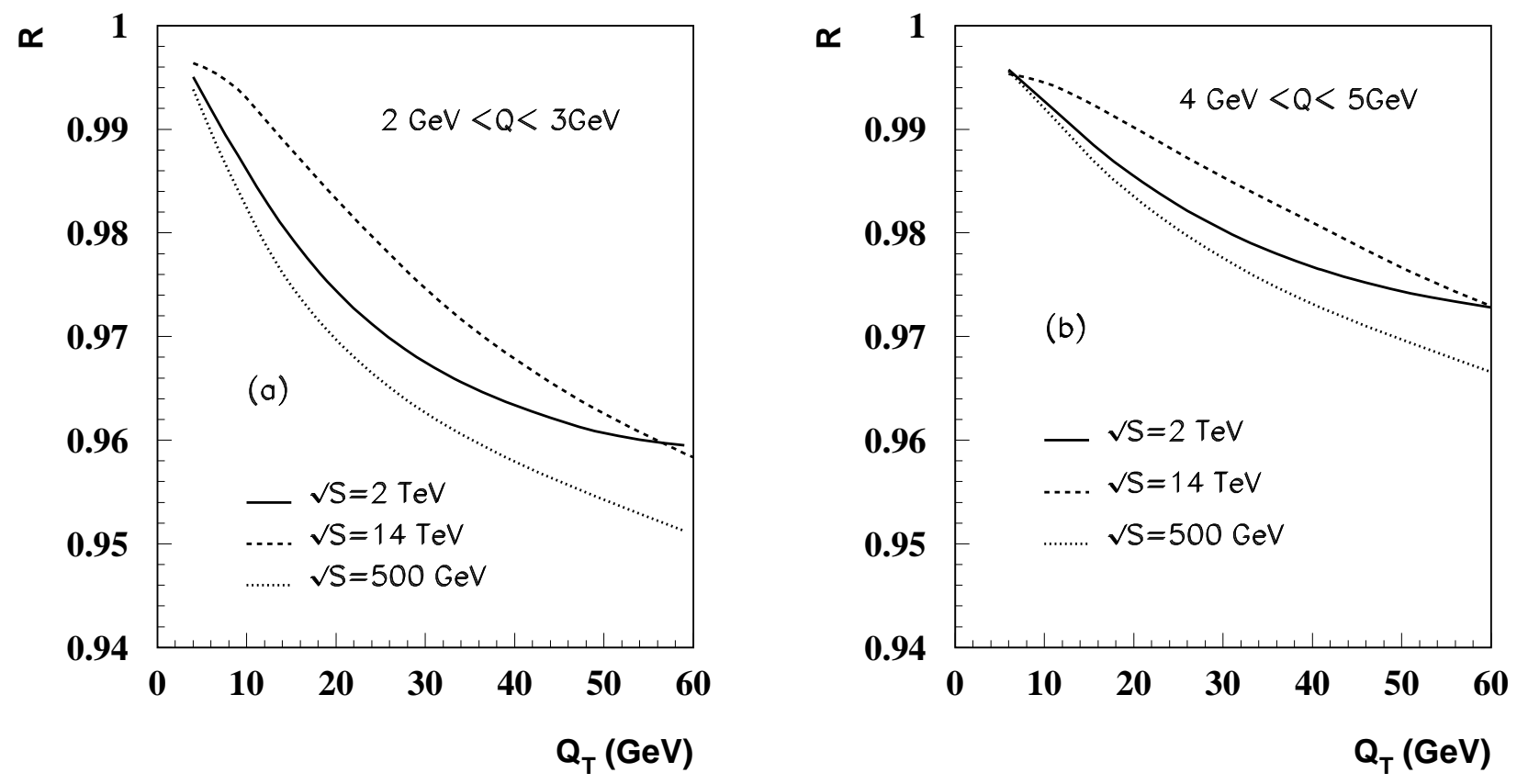

FIG. 13. Ratio $R$ in Eq. (55) as a function of $Q_{T}$ at $y=0$ and mass intervals (a) $2 \leq Q \leq 3 \mathrm{GeV}$ and (b) $4 \leq Q \leq 5 \mathrm{GeV}$. Solid, dashed, and dotted lines are for the Tevatron energy $\sqrt{S}=2.0 \mathrm{TeV}$, the LHC energy $\sqrt{S}=14 \mathrm{TeV}$, and the RHIC proton-proton energy $\sqrt{S}=500 \mathrm{GeV}$, respectively.
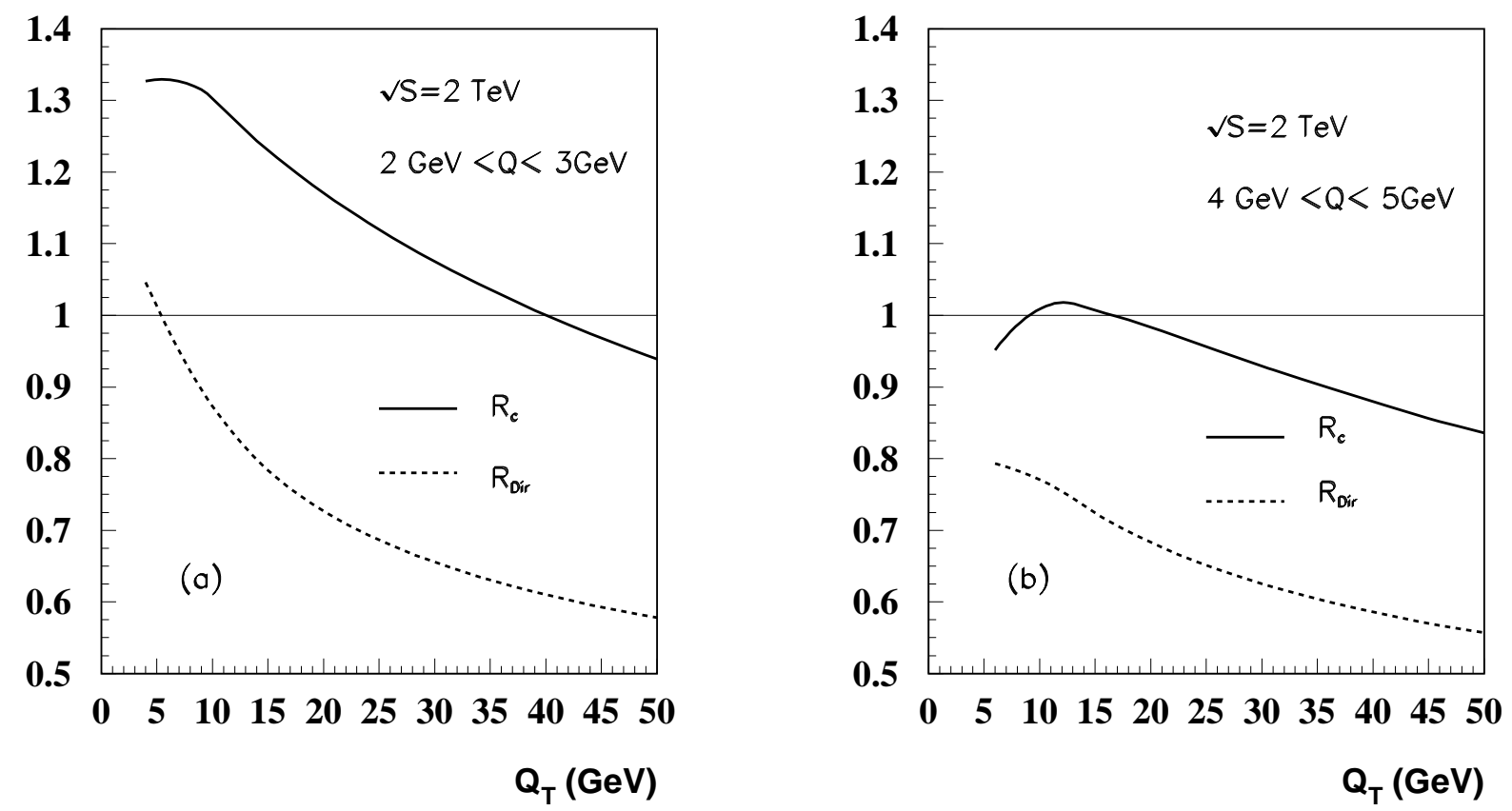

FIG. 14. The ratios in Eqs. (60) and (61) as a function of $Q_{T}$ at $y=0$ and for the Tevatron energy $\sqrt{S}=2.0 \mathrm{TeV}$ and mass intervals (a) $2 \leq Q \leq 3 \mathrm{GeV}$ and (b) $4 \leq Q \leq 5 \mathrm{GeV}$. Solid and dashed lines are for $R_{c}$ and $R_{D i r}$, respectively. 

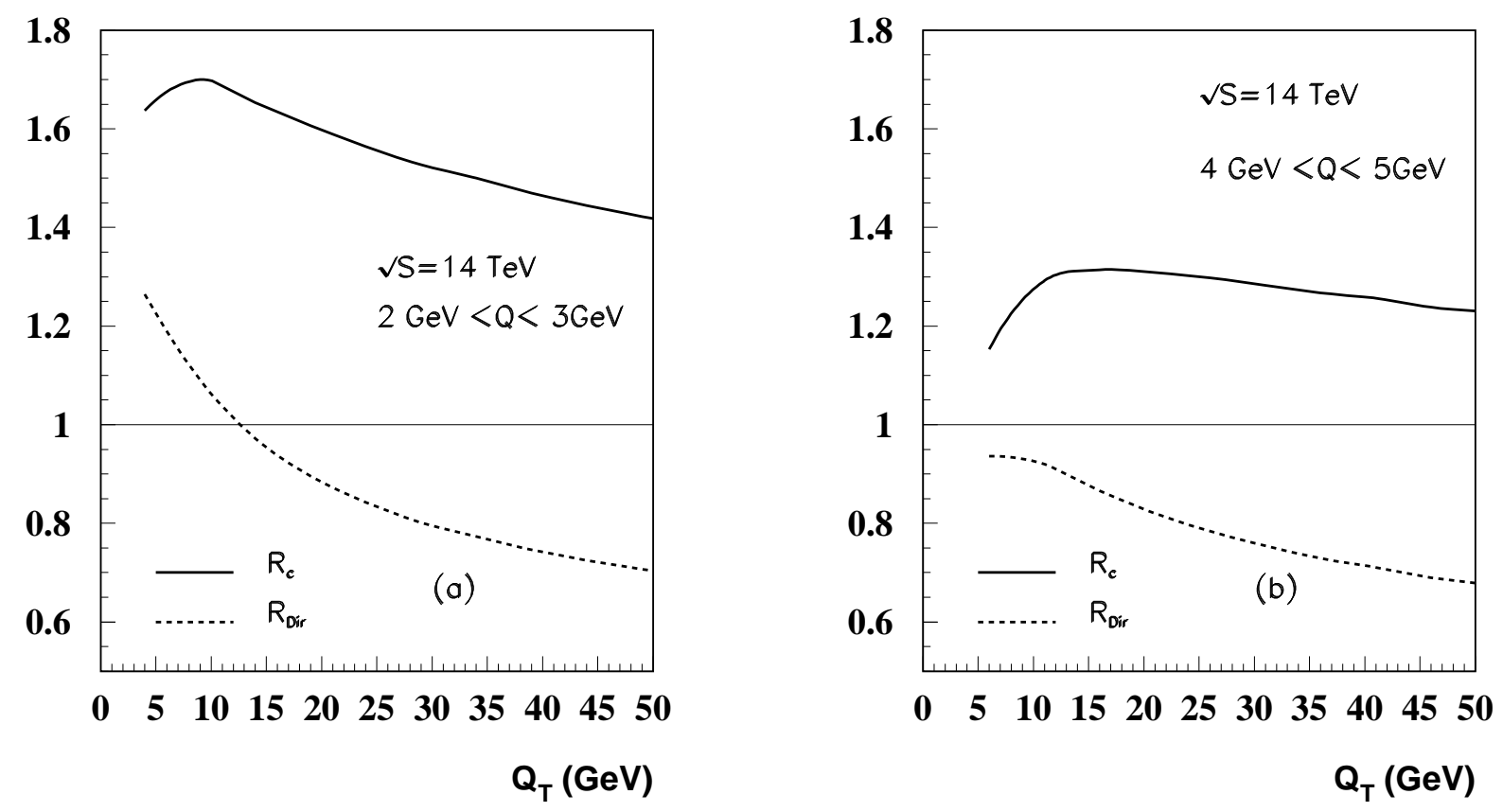

FIG. 15. The ratios in Eqs. (60) and (61) as a function of $Q_{T}$ at $y=0$ for the LHC energy $\sqrt{S}=14 \mathrm{TeV}$ and mass intervals (a) $2 \leq Q \leq 3 \mathrm{GeV}$ and (b) $4 \leq Q \leq 5 \mathrm{GeV}$. Solid and dashed lines are for $R_{c}$ and $R_{D i r}$, respectively.
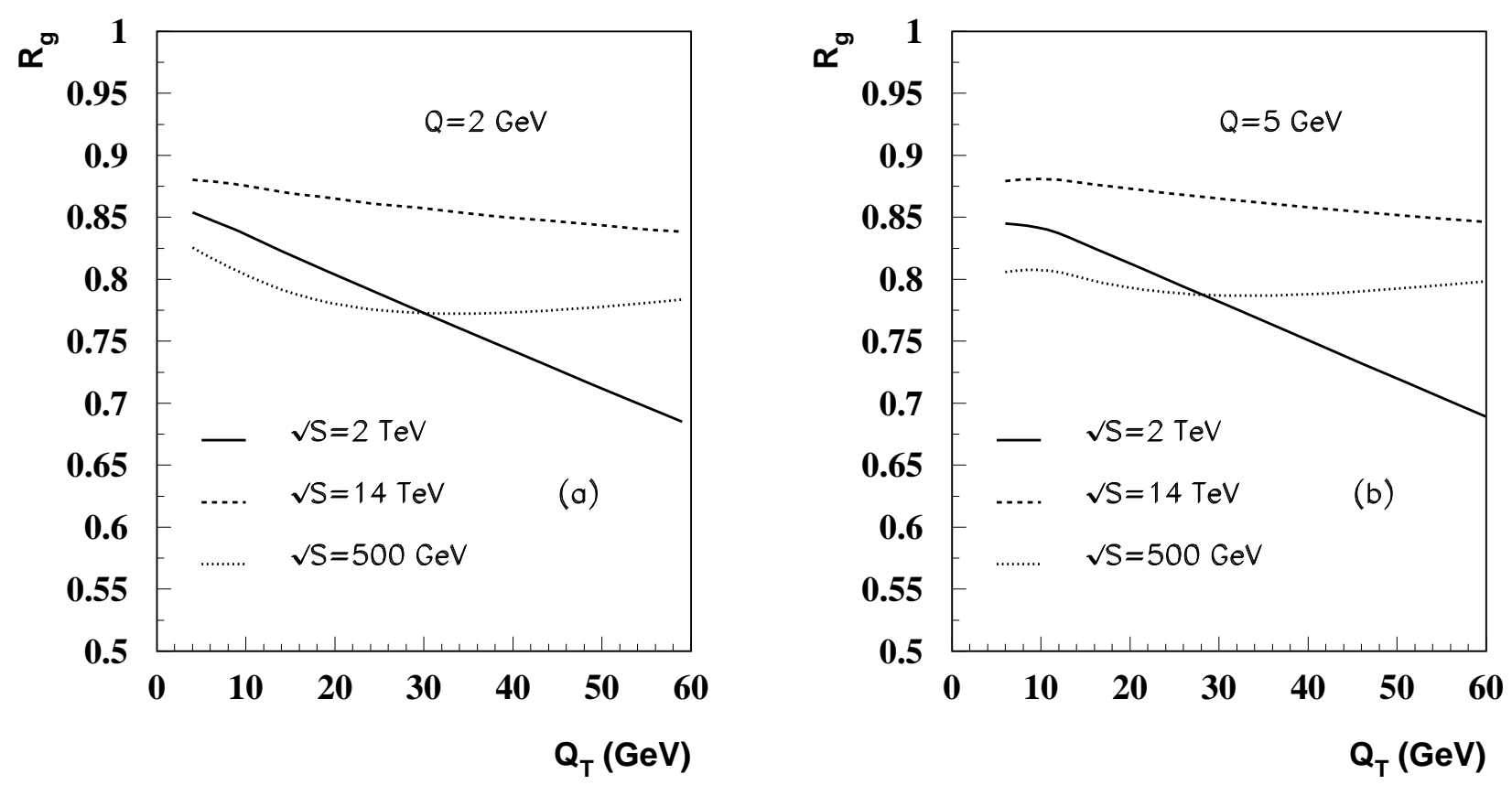

FIG. 16. Ratio $R_{g}$ in Eq. (63) as a function of $Q_{T}$ at $y=0$ at the Tevatron energy (solid), the LHC energy (dashed), and the RHIC proton-proton energy (dotted) for virtual photon invariant masses (a) $Q=2 \mathrm{GeV}$ and (b) $Q=5 \mathrm{GeV}$. 